\title{
Attractors and Finite-Dimensional Behaviour in the 2D Navier-Stokes Equations
}

\author{
James C. Robinson \\ Mathematics Institute, University of Warwick, Coventry CV4 7AL, UK \\ Correspondence should be addressed to James C. Robinson; j.c.robinson@warwick.ac.uk
}

Received 10 March 2013; Accepted 29 April 2013

Academic Editors: I. Fragala, G. Mantica, and A. Peris

Copyright (C) 2013 James C. Robinson. This is an open access article distributed under the Creative Commons Attribution License, which permits unrestricted use, distribution, and reproduction in any medium, provided the original work is properly cited.

\begin{abstract}
The purpose of this review is to give a broad outline of the dynamical systems approach to the two-dimensional Navier-Stokes equations. This example has led to much of the theory of infinite-dimensional dynamical systems, which is now well developed. A second aim of this review is to highlight a selection of interesting open problems, both in the analysis of the two-dimensional Navier-Stokes equations and in the wider field of infinite-dimensional dynamical systems.
\end{abstract}

\section{Introduction}

The Navier-Stokes equations are the fundamental mathematical model of fluid flow; a physical derivation of the equations can be found in Batchelor [1] or Doering and Gibbon [2], for example. Their rigorous analysis goes back to Leray [3], who proved the global existence of weak $\left(L^{2}\right.$-valued) solutions in 3D and local existence of strong $\left(H^{1}\right.$-valued) solutions; similar results were obtained by Hopf [4] for bonded domains. Global existence and uniqueness of weak solutions in the 2D case was first shown by Ladyzhenskaya [5].

The dynamical systems approach to the Navier-Stokes equations was developed over a number of years, notably by Ladyzhenskaya [6] and Foias, Constantin, Temam, and coauthors; see Constantin et al. [7], for example. Delay differential equations provided a stimulus for the development of the theory from a different but related viewpoint; see Hale et al. [8], for example.

Since rigorous existence and uniqueness results are only available for the $2 \mathrm{D}$ equations, we confine ourselves here to this case.

The Navier-Stokes equations are

$$
\frac{\partial u}{\partial t}-v \Delta u+(u \cdot \nabla) u+\nabla p=f(x, t) \quad \nabla \cdot u=0,
$$

posed on a spatial domain $\Omega \subset \mathbb{R}^{2}$, supplemented with appropriate boundary conditions. Here $u$ is the two-component velocity, the parameter $v>0$ is the kinematic viscosity, and $p$ is the scalar pressure, which serves to enforce the divergence-free condition $\nabla \cdot u=0$. The right-hand side is a (somewhat artificial) "body force," which serves to maintain some nontrivial motion of the fluid.

For simplicity we will treat the equations on a periodic domain $\Omega=[0, L]^{2}$, so that $u\left(x+L e_{j}, t\right)=u(x, t)$, where $e_{j}$ are unit vectors parallel to the coordinate axes. In addition we will make the simplifying assumption that $u$ and $f$ have zero average over $\Omega$,

$$
\int_{\Omega} f(x, t) \mathrm{d} x=\int_{\Omega} u(x) \mathrm{d} x=0,
$$

and that $f$ is divergence-free $(\nabla \cdot f=0)$.

Although we will generally confine our analysis to the case of periodic boundary conditions, many results are also true for the case of Dirichlet boundary conditions,

$$
\left.u\right|_{\partial \Omega}=0,
$$

and we will occasionally comment on this case in what follows.

Note that while much of the existence and uniqueness theory, particularly in the 3D case, is carried out in the whole space setting (which allows one to use the tools of harmonic analysis; see e.g., Lemarié-Rieusset [9] or Cannone [10]), this is not convenient in the dynamical systems approach. Even when $f=0$, the decay of solutions to zero is a delicate matter. We discuss this briefly in Section 3 . 
Periodic boundary conditions are particularly useful for analysis, since in this case we can expand $u$ as a Fourier series

$$
u(x)=\sum_{k \in \mathbb{Z}^{2}} \widehat{u}_{k} e^{2 \pi i k \cdot x / L} \quad \text { with } \widehat{u}_{k}=\overline{\widehat{u}}_{-k}, \quad k \cdot \widehat{u}_{k}=0,
$$

where $\bar{u}$ denotes the complex conjugate of $u$ (this condition enforces the reality of $u$ ).

One of the main reasons for the rigorous study of the Navier-Stokes equations is the attempt to gain further understanding of fluid turbulence. However, even at the modelling level there are questions to answer. The standard heuristic model of turbulence (see Frisch [11]) requires the fluid to be subject to the injection of energy at a constant rate at a particular "scale." One might attempt to model this by using a forcing $f=\varepsilon w_{k}$, where $w_{k}$ is some combination of Fourier modes associated with the lengthscale $L / k$. But if one multiplies the equation by $u$ and integrates then the resulting energy equation

$$
\frac{1}{2} \frac{\mathrm{d}}{\mathrm{d} t}\|u\|_{L^{2}}^{2}+v\|D u\|_{L^{2}}^{2}=\varepsilon\left(u, w_{k}\right)
$$

shows that the rate of energy injection (the right-hand side) depends on the velocity field $u$. Of course, one could take a time-dependent forcing

$$
f(t)=\varepsilon \frac{w_{k}}{\left(u, w_{k}\right)}
$$

(in the hope that $\left(u(t), w_{k}\right) \neq 0$ for all $t \geq 0$ ) but this seems very unnatural.

Open Question 1. What is a reasonable model equation for homogeneous fully developed turbulence?

For an interesting example of a nonstandard velocitydependent forcing, see Cheskidov et al. [12].

\section{The Navier-Stokes Equations}

2.1. The Navier-Stokes Equations in Functional Form. We now rewrite the Navier-Stokes equations in a more convenient way. The ideas go back to Leray [3] and are now standardsee, for example, Constantin and Foias [13], Temam [14] or [15], Ladyzhenskaya [5], and Robinson [16].

The main idea is to remove the pressure by projecting onto the space of all divergence-free vector fields; under appropriate boundary conditions gradients and divergencefree functions are orthogonal, since

$$
\int_{\Omega} \nabla p \cdot u \mathrm{~d} x=\int_{\partial \Omega} p u-\int_{\Omega} p(\nabla \cdot u) .
$$

If we denote by $\Pi$ the orthogonal projection in $L^{2}$ onto the space of all such divergence-free functions (the "Leray projector"), then $\Pi \nabla p=0$, and so we obtain an equation for $u$ alone as,

$$
\frac{\partial u}{\partial t}+\Pi[(u \cdot \nabla) u]+v A u=f
$$

where $A=-\Pi \Delta$ is known as the Stokes operator (recall that we assumed that $f$ was divergence-free).

Let

$$
\begin{gathered}
\mathscr{V}=\left\{u \in C^{\infty}(Q): \nabla \cdot u=0, \int_{Q} u(x) \mathrm{d} x=0,\right. \\
u \text { is } \Omega \text {-periodic }\} ;
\end{gathered}
$$

let $H$ be the completion of $\mathscr{V}$ in the norm of $L^{2}(\Omega)$ which we denote by $|\cdot|$, and let $V$ be the completion of $\mathscr{V}$ in the $H^{1}(\Omega)$ norm. (We use $L^{2}(\Omega), H^{k}(\Omega)$ for spaces of scalar or two-component functions interchangeably-what is meant should be clear from the context.) Since we are assuming that $u$ has zero average, we have the Poincaré inequality

$$
\lambda_{1}|u|^{2} \leq|D u|^{2}
$$

(for the definition of $\lambda_{1}$ see later), and so we can (and will) use $|D u|$ for the norm on $V$ (It is common practice in papers on the Navier-Stokes equations to use $|\cdot|$ for the norm in $H$ and $\|$. $\|$ for the norm in $V$. We will not adopt this practice here, preferring $|D u|$ over $\|u\|$ for the sake of clarity.) We denote by $V^{*}$ the dual of $V$, and denote the norm of $f \in V^{*}$ by

$$
\|f\|_{*}:=\sup \{\langle|f, u|\rangle: u \in V \text { with }|\mathrm{D} u|=1\} .
$$

By $D\left(A^{r}\right)$ we denote the domain of $A^{r}$, that is, all those $u$ for which $\left|A^{r} u\right|$ is finite. Note that in the case of periodic boundary conditions this has a simple characterisation as those Fourier series (4) for which

$$
\left|A^{r} u\right|^{2}:=\sum_{k \in \mathbb{Z}^{2}}|k|^{2 r}\left|\widehat{u}_{k}\right|^{2}<\infty
$$

(cf. [15]). Note that $\left|A^{1 / 2} u\right|=|D u|$. (In the case of periodic boundary conditions we have $A u=-\Delta u$ for all $u \in D(A)$; see [13].)

By $\lambda_{j}$ we denote the eigenvalues of $A$, ordered so that $\lambda_{j+1} \geq \lambda_{j}$; we denote the corresponding eigenfunctions by $w_{j}$, so that $A w_{j}=\lambda_{j} w_{j}$. The constant $\lambda_{1}$ in the Poincaré inequality (10) is given by the first eigenvalue $\lambda_{1}=(2 \pi / L)^{2}$.

We now define a bilinear form $B: V \times V \rightarrow V^{*}$ by setting

$$
B(u, v)=\Pi[(u \cdot \nabla) v] .
$$

(One can also proceed in a more roundabout way, defining $b: V \times V \times V \rightarrow \mathbb{R}$ by $b(u, v, w)=\int_{\Omega}[(u \cdot \nabla) v] \cdot w \mathrm{~d} x$ and then defining $B$ via the Riesz Representation Theorem.) The following properties of $B$ will be useful throughout all that follows. First we have two orthogonality properties; in two and three dimensions we have

$$
(B(u, v), v)=0 \quad \forall u \in H, v \in V,
$$

(this follows from an integration by parts and cancellations due to the boundary conditions), and-only for periodic boundary conditions in two dimensions-we also have

$$
(B(u, u), A u)=0 \quad \forall u \in D(A)
$$


(the proof is by expansion, rearrangement, and cancellation of terms using the fact that $u$ is divergence-free).

In our analysis we frequently require inequalities for $(B(u, v), w)$. Rather than listing them now, we will derive them when required, since the ingredients are very simple: the three-exponent Hölder inequality

$$
\|f g h\|_{L^{1}} \leq\|f\|_{L^{r}}\|g\|_{L^{p}}\|h\|_{L^{q}} \frac{1}{r}+\frac{1}{p}+\frac{1}{q}=1,
$$

and the Sobolev embeddings $H^{1} \subset L^{p}$ for any $p$ in 2D. In addition, in 2D we will need Ladyzhenskaya's inequality

$$
\|u\|_{L^{4}} \leq c\|u\|_{L^{2}}^{1 / 2}\|D u\|_{L^{2}}^{1 / 2}
$$

(this follows from interpolating $H^{1 / 2}$ between $L^{2}$ and $H^{1}$ and then using the $2 \mathrm{D}$ embedding $H^{1 / 2} \subset L^{4}$ along with the Poincaré inequality).

With these definitions we can rewrite the Navier-Stokes equations in the functional form

$$
\frac{\mathrm{d} u}{\mathrm{~d} t}+v A u+B(u, u)=f
$$

2.2. Weak Solutions. Suppose that $f \in V^{*}$. If we assume that $u$ is smooth, then we can multiply the equation by $u$, and then using

$$
(A u, u)=|D u|^{2}, \quad(B(u, u), u)=0
$$

we obtain the simple estimate

$$
\begin{aligned}
\frac{1}{2} \frac{\mathrm{d}}{\mathrm{d} t}|u|^{2}+v|D u|^{2} & =(f, u) \leq\|f\|_{*}|D u| \\
& \leq \frac{1}{2 \nu}\|f\|_{*}^{2}+\frac{v}{2}|D u|^{2},
\end{aligned}
$$

which yields the differential inequality

$$
\frac{\mathrm{d}}{\mathrm{d} t}|u|^{2}+v|D u|^{2} \leq \frac{\|f\|_{*}^{2}}{v},
$$

from which we derive the energy inequality

$$
|u(t)|^{2}+v \int_{0}^{t}|D u(s)|^{2} \mathrm{~d} s \leq\left|u_{0}\right|^{2}+t \frac{\|f\|_{*}^{2}}{v} .
$$

These formal calculations show that one would expect solutions to satisfy

$$
u \in L^{\infty}(0, T ; H) \cap L^{2}(0, T ; V) .
$$

Indeed, this essentially yields the definition of a weak solution.

Definition 1. A weak solution of the Navier-Stokes equations is a function $u \in L^{\infty}(0, T ; H) \cap L^{2}(0, T ; V)$ such that (18) holds as an equality in $V^{*}$ for almost every $t \in[0, T]$.
Note that in the $2 \mathrm{D}$ case

$$
\begin{aligned}
|(B(u, u), v)| & =|(B(u, v), u)| \\
& \leq\|u\|_{L^{4}}^{2}\|D v\|_{L^{2}} \\
& \leq\|u\|_{L^{2}}\|D u\|_{L^{2}}\|D v\|_{L^{2}},
\end{aligned}
$$

using the 2D Ladyzhenskaya inequality $\|u\|_{L^{4}}^{2} \leq\|u\|_{L^{2}}\|D u\|_{L^{2}}$. It follows, since $u \in L^{\infty}(0, T ; H) \cap L^{2}(0, T ; V)$, that we have $B(u, u) \in L^{2}\left(0, T ; V^{*}\right)$, and, hence, that if a weak solution exists, $u_{t} \in L^{2}\left(0, T ; V^{*}\right)$.

For a rigorous proof of the existence of weak solutions one replaces (18) by the Galerkin approximations

$$
\frac{\mathrm{d} u_{n}}{\mathrm{~d} t}+v A u_{n}+P_{n} B\left(u_{n}, u_{n}\right) \quad u_{n}(0)=P_{n} u_{0}
$$

where $P_{n}$ is the orthogonal projection onto the first $n$ eigenfunctions of $A$,

$$
P_{n} u=\sum_{j=1}^{n}\left(u, w_{j}\right) w_{j}
$$

These approximations are essentially finite-dimensional systems of Lipschitz ordinary differential equations and so have solutions that exist while their norm stays finite. By repeating the previous calculations for (25), which are now rigorously justifiable since $u_{n}$ is smooth, one can find uniform bounds on $u_{n}$ in $L^{\infty}(0, T ; H) \cap L^{2}(0, T ; V)$. Similarly, $\mathrm{d} u_{n} / \mathrm{d} t$ is uniformly bounded in $L^{2}\left(0, T ; V^{*}\right)$. Using all these uniform bounds one can extract a subsequence that converges to some $u$ sufficiently strongly that every term in (25) also converges to the required term from the full equation. For details see Constantin and Foias [13] Robinson [16], or Temam [15], for example.

We state formally a result about the existence and uniqueness of weak solutions in the $2 \mathrm{D}$ case. In $3 \mathrm{D}$ weak solutions are known to exist (following the same argument), but their time derivative is less regular $\left[u_{t} \in L^{4 / 3}\left(0, T ; V^{*}\right)\right]$ and continuity into $H$ and uniqueness are not known. It is also not known if every $3 \mathrm{D}$ weak solution must satisfy the energy inequality (22).

Theorem 2. If $f \in V^{*}$ and $u_{0} \in H$, the $2 D$ Navier-Stokes equations have a unique weak solution that exists for all $t \geq 0$; for any $T>0$

$$
u \in L^{\infty}(0, T ; H) \cap L^{2}(0, T ; V), \quad \frac{\mathrm{d} u}{\mathrm{~d} t} \in L^{2}\left(0, T ; V^{*}\right),
$$

and consequently $u \in C^{0}([0, T] ; H)$. All such solutions satisfy the energy inequality (22).

Proof. We have sketched the proof of existence of weak solutions. It remains to prove the continuity into $H$ and uniqueness of the solution. Both rely on the regularity of $u_{t}$. 
The bounds on $u$ in $L^{2}(0, T ; V)$ and on $u_{t}$ in $L^{2}\left(0, T ; V^{*}\right)$ are sufficient to guarantee that $u$ is continuous into $H$; essentially this is enough regularity to ensure that

$$
\frac{\mathrm{d}}{\mathrm{d} t}|u|^{2}=\left\langle u_{t}, u\right\rangle
$$

which on integrating yields continuity of $u$ into $H$ (see e.g., Evans [17] or Robinson [16]). So any weak solution (in 2D) will also be continuous into $H$, that is, will trace out a meaningful trajectory in the energy space.

It remains to prove the uniqueness. To do this, consider two solutions $u$ and $v$ and their difference $w=u-v$. Then the equation for $w$ is

$$
\frac{\mathrm{d} w}{\mathrm{~d} t}+v A w+B(u, w)+B(w, v)=0
$$

Since $w_{t} \in L^{2}\left(0, T ; V^{*}\right)$ and $w \in L^{2}(0, T ; V)$, there is enough regularity to take the inner product with $w$ and obtain (recalling that $(B(u, w), w)=0)$

$$
\begin{aligned}
\frac{1}{2} \frac{\mathrm{d}}{\mathrm{d} t}|w|^{2}+v|D w|^{2} & \leq|(B(w, v), w)| \\
& \leq\|w\|_{L^{4}}^{2}|D v| \\
& \leq c|w||D w||D v| .
\end{aligned}
$$

After an application of Young's inequality this yields

$$
\frac{\mathrm{d}}{\mathrm{d} t}|w|^{2}+\nu|D w|^{2} \leq \frac{c}{v}|w|^{2}|D v|^{2}
$$

Thus

$$
|w(t)|^{2} \leq|w(0)|^{2} \exp \left(\frac{c}{v} \int_{0}^{t}|D v(s)|^{2} \mathrm{~d} s\right)
$$

in particular if $w(0)=0$ then $w(t)=0$ for all $t \geq 0$, since $v \in L^{2}(0, T ; V)$.

2.3. Strong Solutions and Regularisation. We now suppose that $f \in H$. We show that if $u_{0} \in V$ then we obtain a more regular type of solution and deduce that if $u_{0} \in H$ then $u(t) \in V$ for every $t>0$.

Suppose that we now take the inner product of (18) with $A u$. Since we are working with periodic boundary conditions we can use the orthogonality property $(B(u, u), A u)=0$ to make our analysis a little easier, and we obtain

$$
\frac{1}{2} \frac{\mathrm{d}}{\mathrm{d} t}|D u|^{2}+\nu|A u|^{2}=(f, A u) \leq|f||A u|,
$$

which after an application of Young's inequality yields

$$
\frac{\mathrm{d}}{\mathrm{d} t}|D u|^{2}+\nu|A u|^{2} \leq \frac{1}{v}|f|^{2}
$$

An integration in time shows that

$$
|D u(t)|^{2}+v \int_{0}^{t}|A u(s)|^{2} \mathrm{~d} s \leq\left|D u_{0}\right|^{2}+\frac{t}{v}|f|^{2}
$$

provided that $u_{0} \in V$ the solution satisfies

$$
u \in L^{\infty}(0, T ; V) \cap L^{2}(0, T ; D(A)) .
$$

A strong solution is a weak solution that has this additional regularity.

Proposition 3. If $f \in H$ and $u_{0} \in V$ then there exists a unique strong solution such that for any $T>0$

$$
u \in L^{\infty}(0, T ; V) \cap L^{2}(0, T ; D(A)) .
$$

In addition $u_{t} \in L^{2}(0, T ; H)$ and $u \in C^{0}([0, T] ; V)$.

We can deduce from this result that weak solutions immediately regularise, that is, become strong.

Corollary 4. If $f \in H$ and $u_{0} \in H$, then $u(t) \in V$ for all $t>0$.

Proof. Let $u$ be the weak solution of (18), and fix some $T>0$. Since $u \in L^{2}(0, T ; V)$, there exists an $h<T$ such that $u(h) \in$ $V$. The solution $u_{h}(\cdot)$ of the NSE with $u_{h}(0)=u(h)$ is strong, and so $u_{h} \in C^{0}([0, T-h], V)$. But the function $u(\cdot-h)$ is a weak solution of (18); by uniqueness it is the solution with this initial data. Thus $u(T)=u_{h}(T-h) \in V$ as claimed.

For an alternative and perhaps more elegant proof one can take the inner product of (18) with $t A u$ and follow the previous calculations above. This yields more information, including the rate of regularisation of the $V$ norm (like $t^{-1 / 2}$ ) as follows:

$$
\begin{aligned}
t|D u(t)|^{2}+v \int_{0}^{t} s|A u(s)|^{2} \mathrm{~d} s \\
\leq \frac{t^{2}}{2 v}|f|^{2}+\int_{0}^{t}|D u(s)|^{2} \mathrm{~d} s \\
\leq \frac{t^{2}}{2 v}|f|^{2}+\frac{t}{v^{2}}\|f\|_{*}^{2}+\frac{\left|u_{0}\right|^{2}}{v},
\end{aligned}
$$

using (22).

We note that in 3D one can only prove the local existence of strong solutions; that is, solutions remain bounded in $V$ on some time interval $[0, T)$, where $T$ depends on $\left|D u_{0}\right|$; these are unique in the class of weak solutions satisfying the energy inequality (22).

Open Question 2 (Clay Millennium Problem). Do the threedimensional Navier-Stokes equations have strong solutions for all $t \geq 0$ ?

2.4. The Solution Semigroup. We define a dynamical system using the Navier-Stokes equations on the phase space $H$, although we could also use $V$ or indeed $D\left(A^{k / 2}\right)$ for any $k \in \mathbb{N}$. Given an initial condition $u_{0} \in H$, we have seen that the equation has a unique solution $u\left(t ; u_{0}\right)$ for all positive times. In this case, we can define a $C^{0}$ semigroup of solution operators $S(t): H \rightarrow H$, for $t \geq 0$, by

$$
S(t) u_{0}=u\left(t ; u_{0}\right)
$$


These operators satisfy

$$
\begin{aligned}
& S(0)=\mathrm{I}, \\
& S(t) S(s)=S(s) S(t)=S(s+t), \quad t, s \geq 0, \\
& S(t) x_{0} \text { is continuous in } x_{0} \text { and } t,
\end{aligned}
$$

and we can consider the semidynamical system

$$
\left(H,\{S(t)\}_{t \geq 0}\right) \text {. }
$$

We define the semigroup only for $t \geq 0$ since, as for many parabolic PDEs, it is unnatural to consider $t<0$. For example, backwards solutions of the heat equation $\left(u_{t}=\Delta u\right)$ can blow up instantaneously unless the initial data is analytic.

2.5. Backwards Uniqueness. We cannot solve the equations backwards, but we can show that solutions enjoy the backwards uniqueness property, that is, that $S(t): H \rightarrow H$ is injective for every $t>0$ :

$$
S(t) u_{0}=S(t) v_{0} \Longrightarrow u_{0}=v_{0} .
$$

We do this by combining the argument in Temam ([18], Chapter III Section 6) which shows that $S(t): V \rightarrow V$ is injective, and an observation in Chapter 11 of Constantin and Foias [13].

First we prove backwards uniqueness for an abstract problem when the difference $w$ of two solutions satisfies

$$
\frac{\mathrm{d} w}{\mathrm{~d} t}+A w=h(t, w)
$$

where for $t>0$

$$
|h(t, w)| \leq k(t)\left|A^{1 / 2} w\right|
$$

for some $k \in L^{2}(0, T)$.

The proof uses the "Dirichlet quotient"

$$
\Lambda(w)=\frac{\left|A^{1 / 2} w\right|^{2}}{|w|^{2}}
$$

By differentiating the expression for $\Lambda$, substituting for $w_{t}$, using (43), and observing that $(A w-\Lambda w, w)=0$ (see Lemma III.6.1 in [18]), one can deduce that

$$
\frac{\mathrm{d} \Lambda}{\mathrm{d} t} \leq 2 k^{2} \Lambda
$$

from which it follows that

$$
\Lambda(t) \leq \Lambda(0) \exp \left(\int_{0}^{t} 2 k^{2}(s) \mathrm{d} s\right) .
$$

Lemma 5. If $w \in L^{\infty}(0, T ; V) \cap L^{2}(0, T ; D(A))$ and $w(T)=0$ for some $T>0$, then $w(t)=0$ for all $0 \leq t \leq T$.

Proof. For a contradiction assume that $\left|w\left(t_{0}\right)\right| \neq 0$ for some $t_{0} \in[0, T)$. Then since $w(t)$ is continuous into $L^{2}$, there exists an $\epsilon>0$ such that $w(t) \neq 0$ on $\left[t_{0}, t_{0}+\epsilon\right)$. Let $t_{1} \leq T$ be the largest time such that $w(t) \neq 0$ on $\left[t_{0}, t_{1}\right)$, and note that $w\left(t_{1}\right)=0$.
On $\left[t_{0}, t_{1}\right)$ we have

$$
\begin{aligned}
\frac{\mathrm{d}}{\mathrm{d} t} \log \frac{1}{|w|} & =-\frac{1}{2} \frac{\mathrm{d}}{\mathrm{d} t} \log |w|^{2}=\frac{\left(-w^{\prime}, w\right)}{|w|^{2}} \\
& =-\frac{(h-A w, w)}{|w|^{2}}=\Lambda-\frac{(h, w)}{|w|^{2}} \\
& \leq \Lambda+k \Lambda^{1 / 2} \leq 2 \Lambda+k^{2} .
\end{aligned}
$$

Integrating between $t_{0}$ and $t \in\left[t_{0}, t_{1}\right)$ yields

$$
\begin{aligned}
\log \frac{1}{|w(t)|} & \leq \log \frac{1}{\left|w\left(t_{0}\right)\right|}+\int_{t_{0}}^{t} 2 \Lambda(s)+k^{2}(s) \mathrm{d} s \\
& \leq \log \frac{1}{\left|w\left(t_{0}\right)\right|}+\int_{0}^{T} 2 \Lambda(s)+k^{2}(s) \mathrm{d} s
\end{aligned}
$$

whence $1 /|w(t)|$ is bounded as $t \uparrow t_{1}$, a contradiction.

This argument has been extended by Kukavica [19] to allow for additional logarithmic terms in the estimate (44).

Corollary 6. The semigroup $S(t): H \rightarrow H$ is injective.

Proof. Suppose that $u_{0}, v_{0} \in H$ and $u(T)=v(T)$. Since $S(\cdot)$ has a regularising effect (Corollary 4 ), for any $\delta>0$ we know that $u_{\delta}=S(\delta) u_{0}$ and $v_{\delta}=S(\delta) v_{0}$ are elements of $V$. It follows from Proposition 3 that $u_{\delta}(t):=S(t) u_{\delta}$ and $v_{\delta}(t):=S(t) v_{\delta}$ are elements of $L^{\infty}(0, T-\delta ; V) \cap L^{2}(0, T-\delta ; D(A))$.

If $w=u_{\delta}-v_{\delta}$ then

$$
w_{t}+A w+B\left(u_{\delta}, w\right)+B\left(w, v_{\delta}\right)=0
$$

Noting that

$$
\begin{aligned}
& \left|B\left(u_{\delta}, w\right)+B\left(w, v_{\delta}\right)\right| \\
& \quad \leq c\left(\left\|u_{\delta}\right\|_{L^{\infty}}\left|A^{1 / 2} w\right|+\|w\|_{L^{4}}\left\|D v_{\delta}\right\|_{L^{4}}\right) \\
& \quad \leq c\left(\left|A u_{\delta}\right|+\left|A v_{\delta}\right|\right)\left|A^{1 / 2} w\right|
\end{aligned}
$$

it follows that $F(w):=B\left(u_{\delta}, w\right)+B\left(w, v_{\delta}\right)$ satisfies the condition of Lemma 5 , and, hence, that $u_{\delta}(0)=v_{\delta}(0)$, that is, $u(\delta)=$ $v(\delta)$ for every $\delta>0$.

Finally we simply observe that $u$ and $v$ are continuous from $[0, T]$ into $H$, and it follows that $u_{0}=v_{0}$.

2.6. The Pressure. It is easy to start to think of the "NavierStokes equations" as the equations in their functional form (18) and, hence, of the velocity as the only dependent variable of interest. Although this is a mathematically convenient point of view, it is often the pressure that is of interest in physical problems.

Thus one must ask whether $p$ can be recovered given $u$. If we take the divergence of (1) then we obtain a Poisson equation for the pressure

$$
\Delta p=-\nabla \cdot[(u \cdot \nabla) u]
$$

(we took $\nabla \cdot f=0$ ). If we impose the additional condition that $\int_{Q} p=0$, then this equation has a unique solution 
(see Simon [20]). On $\mathbb{R}^{2}$ or $\mathbb{T}^{2}$ one can in fact obtain bounds on the pressure in any Lebesgue space

$$
\|p\|_{L^{r}} \leq c_{r}\|u\|_{L^{2 r}}^{2}
$$

This follows since $p=R_{i} R_{j}\left(u_{i} u_{j}\right)$, where $R_{i}$ denotes the Riesz transform in the $i$ th component, using the CalderonZygmund Theorem (see [21], e.g., for the whole space and [22] for its periodic variant).

Open Question 3. Does the pressure estimate (53) hold in bounded domains?

One could also wonder about the converse question: does the pressure determine the velocity? In a two-dimensional incompressible flow this seems at least plausible; one can determine the velocity by a single scalar streamfunction, and the pressure is a single scalar. To formalise this, we write

$$
u=\left(\phi_{y},-\phi_{x}\right)
$$

and then the pressure equation (52) becomes

$$
\begin{aligned}
\Delta p= & -\nabla \cdot\left[\left(\phi_{y} \partial_{x}-\phi_{x} \partial_{y}\right)\left(\phi_{y},-\phi_{x}\right)\right] \\
= & -\nabla \cdot\left(\phi_{y} \phi_{x y}-\phi_{x} \phi_{y y},-\phi_{y} \phi_{x x}+\phi_{x} \phi_{x y}\right) \\
= & -\phi_{x y}^{2}-\phi_{y} \phi_{x x y}+\phi_{x x} \phi_{y y}+\phi_{x} \phi_{y y x} \\
& +\phi_{x x} \phi_{y y}+\phi_{y} \phi_{x x y}-\phi_{x y}^{2}-\phi_{x} \phi_{x y y} \\
= & \phi_{x x} \phi_{y y}-\phi_{x y}^{2} ;
\end{aligned}
$$

that is,

$$
\phi_{x x} \phi_{y y}-\phi_{x y}^{2}=\Delta p .
$$

Thus the stream function $\phi$ satisfies the Monge-Ampère equation ( $\operatorname{det} H(\phi)=\Delta p$, where $H(\phi)$ denotes the Hessian of $\phi$ ), as was observed by Larchevêque [23]. This equation is well understood when the right-hand side is positive, since in this case the equation provides a canonical example of a "nonlinear elliptic problem," see Chapter 11 of Gilbarg and Trudinger [24], for example. However, little is known when the right-hand side changes sign since in this case the problem is of mixed (nonlinear) elliptic/hyperbolic type.

Open Question 4 (Gero Friesecke). For 2D incompressible flow with periodic (or Dirichlet) boundary conditions, does the pressure determine the velocity uniquely?

If so one could reduce the $2 \mathrm{D}$ Navier-Stokes dynamical system to a dynamical system for the scalar $p$.

\section{3. "Trivial Dynamics" When $f=0$}

When the forcing is zero all solutions decay to zero and the asymptotic dynamics are trivial. This is simple to show on any domain in which the Poincaré inequality (10) is valid, in particular on a bounded or periodic domain (with the zero average condition on $f$ and $u$ ). Indeed, from (21) we have

$$
\frac{\mathrm{d}}{\mathrm{d} t}|u|^{2} \leq-\nu|D u|^{2} \leq-\nu \lambda_{1}|u|^{2},
$$

which shows that $u$ converges to zero (in $L^{2}$ ) exponentially fast. A more detailed analysis due to Foias and Saut [25], shows that in fact

$$
\frac{|D u(t)|}{|u(t)|} \longrightarrow \Lambda \text { as } t \rightarrow \infty
$$

where $\Lambda$ is an eigenvalue of the Stokes operator, and

$$
\lim _{t \rightarrow \infty} e^{\nu \Lambda t} u(t)=u_{\Lambda}
$$

where $u_{\Lambda}$ is an eigenfunction of the Stokes operator with eigenvalue $\Lambda$.

On the whole space the analysis is significantly more involved. Following Schonbek [26] (who considered the harder problem of decay of weak solutions in $\mathbb{R}^{3}$ ) one can consider the energy equation in Fourier space

$$
\begin{aligned}
\frac{1}{2} \frac{\mathrm{d}}{\mathrm{d} t} \int_{\mathbb{R}^{n}}|\widehat{u}|^{2} \mathrm{~d} \xi & \leq-\int_{\mathbb{R}^{n}}|\xi|^{2}|\widehat{u}|^{2} \mathrm{~d} \xi \\
& \leq-\int_{|\xi| \leq r(t)}|\xi|^{2}|\widehat{u}|^{2} \mathrm{~d} \xi-\int_{|\xi| \geq r(t)}|\xi|^{2}|\widehat{u}|^{2} \mathrm{~d} \xi
\end{aligned}
$$

where $r(t)=(t+1)^{-1 / 2}$. This "Fourier splitting" technique allows one to estimate both integrals, using the fact that $|\widehat{u}(\xi, t)| \leq C|\xi|^{-1}$ for $|\xi| \leq r(t)$, and thus deduce that

$$
|u(t)| \leq C(t+1)^{-1 / 2} .
$$

In the two-dimensional case Gallay and Wayne [27] were able to go much further and recover a whole-space version of the results of Foias and Saut. By considering the equation for the vorticity $\omega=\nabla \wedge u$ and making the change of variables

$$
\xi=\frac{x}{\sqrt{1+t}} \quad \tau=\log (1+t)
$$

the linear part of the equation becomes the Schrödinger operator

$$
\Delta_{\xi} w+\frac{1}{2}\left(\xi \cdot \nabla_{\xi}\right) w+w
$$

whose spectral properties are well understood. As a consequence, they could show that the solutions decay to zero like a particular solution, the Oseen vortex, with a magnitude depending on the total initial vorticity $\int_{\mathbb{R}^{2}} \omega \mathrm{d} x$.

Thus when $f=0$ solutions all decay to zero. It seems reasonable to claim that $u=0$ is the "attractor" of the dynamical system $S(t)$ in this case. We now make this notion more precise and then examine the more interesting case when $f \neq 0$. 


\section{The Global Attractor}

We now leave the Navier-Stokes equations for a time and give the definition of the global attractor for an abstract semiflow on a Hilbert space $H$ and prove some properties that all such attractors share. General treatments of the theory of attractors are given in Babin and Vishik [28], Chepyzhov and Vishik [29], Chueshov [30], Hale [31], Ladyzhenskaya [6], Robinson [16], Temam [18], and Vishik [32].

4.1. Existence of the Global Attractor. We say that a set $B \subset H$ attracts $X \subset H$ if

$$
\operatorname{dist}(S(t) X, B) \longrightarrow 0 \text { as } t \longrightarrow \infty \text {, }
$$

where $\operatorname{dist}(X, Y)$ is the Hausdorff semidistance between two sets,

$$
\operatorname{dist}(X, Y)=\operatorname{supinf}_{x \in X}|x-y| .
$$

Note that this distance does not define a metric-indeed, if $\operatorname{dist}(X, Y)=0$ then one only has $X \subset Y$. To obtain a metric on subsets of $H$, we need to use the symmetric Hausdorff distance

$$
\operatorname{dist}_{\mathscr{H}}(X, Y)=\max (\operatorname{dist}(X, Y), \operatorname{dist}(Y, X)) .
$$

Definition 7. The global attractor $\mathscr{A}$ is the maximal compact invariant set,

$$
S(t) \mathscr{A}=\mathscr{A} \quad \forall t \geq 0,
$$

that attracts all bounded sets (is "attracting"):

$$
\operatorname{dist}(S(t) X, \mathscr{A}) \longrightarrow 0 \quad \text { as } t \longrightarrow \infty \text {, }
$$

for any bounded set $X \subset H$.

That $\mathscr{A}$ is maximal means that if $Y$ is a bounded invariant set then $\mathscr{A} \supset Y$. Equation (68) says that $\mathscr{A}$ attracts all orbits, at a rate uniform on any bounded set. Without the compactness condition we could just take $\mathscr{A}=H$. Note that while $\mathscr{A}$ is the maximal compact invariant set, it is also the minimal set that attracts all bounded sets (the proofs are simple). Confusion is possible, since various authors refer to $\mathscr{A}$ as the "minimal attractor" and others as the "maximal attractor."

We give a result on the existence of global attractors in a version inspired by a similar result due to Crauel [33] for random dynamical systems; see also Hale [31] and Babin and Visihik [28]. (The first result along these lines seems to be due to Billotti and LaSalle [34].)

First we define the $\omega$-limit set of a set $X$, which consists of all the limit points of the orbit of $X$ as follows:

$$
\omega(X)=\left\{y: \exists t_{n} \longrightarrow \infty, x_{n} \in X \text { with } S\left(t_{n}\right) x_{n} \longrightarrow y\right\} .
$$

This can be also be characterised as

$$
\omega(X)=\bigcap_{t \geq 0} \bar{S} S(s) X .
$$

$\omega(X)$ in some sense captures all the recurrent dynamics of the orbit through $X$.
Theorem 8. A semigroup $S(t)$ has a global attractor $\mathscr{A}$ if and only if it has a compact attracting set $K$, and then $\mathscr{A}=\omega(K)$.

The proof requires the following simple lemma.

Lemma 9. If $K$ is a compact set and $x_{n}$ is a sequence such that

$$
\operatorname{dist}\left(x_{n}, K\right) \longrightarrow 0
$$

then $\left\{x_{n}\right\}$ has a convergent subsequence whose limit lies in $K$.

Proof. For each $x_{n}$ find a point $k_{n} \in K$ such that $\left|x_{n}-k_{n}\right|=$ $\operatorname{dist}\left(x_{n}, K\right)$. Since $K$ is compact there is a subsequence $k_{n_{j}} \rightarrow$ $k \in H$, and it follows that $x_{n_{j}} \rightarrow k$.

As a first step to proving Theorem 8 we prove the following properties of $\omega$-limit sets.

Proposition 10. If there exists a compact attracting set $K$ then the $\omega$-limit set $\omega(X)$ of any bounded set $X$ is a nonempty, invariant, closed subset of $K$. Furthermore $\omega(X)$ attracts $X$.

Proof. To see that $\omega(X)$ is nonempty choose some point $x \in$ $X$. Then since $K$ is attracting

$$
\operatorname{dist}(S(n) x, K) \longrightarrow 0 \text {. }
$$

It follows that for some sequence $n_{j} \rightarrow 0$

$$
S\left(n_{j}\right) x \longrightarrow x^{*} \in K .
$$

As the intersection of a decreasing sequence of closed sets, $\omega(X)$ is clearly closed. To show that $\omega(X) \subset K$ suppose that $t_{n} \rightarrow \infty, x_{n} \in X$ and

$$
S\left(t_{n}\right) x_{n} \longrightarrow y .
$$

Then since $K$ is attracting

$$
\operatorname{dist}\left(S\left(t_{n}\right) x_{n}, K\right) \longrightarrow 0,
$$

implying that a subsequence of $S\left(t_{n}\right) x_{n}$ converges to a point in $K$. Since the sequence itself converges it follows that $y \in K$. So $\omega(X)$ is compact.

Now suppose that $\omega(X)$ does not attract $X$. Then there exists a $\delta>0$ and a sequence of $t_{n}$ such that

$$
\operatorname{dist}\left(S\left(t_{n}\right) X, \omega(X)\right)>\delta,
$$

and, hence, $x_{n} \in X$ such that

$$
\operatorname{dist}\left(S\left(t_{n}\right) x_{n}, \omega(X)\right)>\delta .
$$

However, the previous argument shows that a subsequence of $\left\{S\left(t_{n}\right) x_{n}\right\}$ converges to some point $z$. By (77) we should have

$$
\operatorname{dist}(z, \omega(X)) \geq \delta,
$$

while by definition $z \in \omega(X)$. So $\omega(X)$ attracts $X$. 
Now observe that

$$
A \subseteq B \Longrightarrow \omega(A) \subseteq \omega(B),
$$

and that since $\omega(X)$ is invariant

$$
\omega[\omega(X)]=\bigcap_{t \geq 0} \overline{\bigcup_{s \geq t} S(s) \omega X}=\omega(X) .
$$

Proof of Theorem 8. It follows from the previous proposition that $\omega(K)$ is nonempty, compact, invariant, and attracts $K$. So all we have to prove is that $\omega(K)$ attracts $X$. Since $\omega(X)$ attracts $X$ it suffices to show that $\omega(X) \subset \omega(K)$. But this follows immediately from (79) and (80). The "only if" part is clear, taking $K=\mathscr{A}$.

In order to apply Theorem 8 we often prove something stronger than the existence of a compact attracting set, namely the existence of a compact absorbing set. We say that a set $K$ absorbs $B$ if there is a time $t_{0}(B)$ such that

$$
S(t) B \subset K \quad \forall t \geq t_{0}(B) .
$$

We say that $K$ is absorbing in $H$ if it absorbs every bounded subset $B$ of $H$.

4.2. Structure of the Attractor. We now want to examine the attractor itself in more detail. We show that it is connected, consists of all complete bounded orbits, and contains the unstable manifolds of all fixed points and periodic orbits. This gives us a better idea of the kind of dynamics we can expect to understand if we restrict our attention to the attractor.

\subsubsection{The Global Attractor is Connected}

Proposition 11. If $\mathscr{A}$ is the global attractor of a semigroup $S(\cdot)$ on a Hilbert space $H$ then $\mathscr{A}$ is connected.

Proof. If $\mathscr{A}$ is not connected then $\mathscr{A}$ is the disjoint union of two nonempty compact sets $A_{1}$ and $A_{2}$, which are therefore separated by a distance $2 \delta$. Let $B$ be a ball that contains $\mathscr{A}$; since $\mathscr{A}$ attracts $B$ there exists a $t_{0}$ such that $\operatorname{dist}(S(t) B, \mathscr{A})<$ $\delta / 2$ for all $t \geq t_{0}$. Since $B$ is connected so is $S(t) B$, from which it follows that either $\operatorname{dist}\left(S(t) B, A_{1}\right)<\delta$ or $\operatorname{dist}\left(S(t) B, A_{2}\right)<$ $\delta$ for all $t \geq t_{0}$. This contradicts the fact that both $A_{1}$ and $A_{2}$ are nonempty.

In general it is not possible to show that the attractor is path connected (two points can be joined by a curve); there are (perhaps artificial) examples of sets that are not path connected that can be global attractors. Günther and Segal [35] remark that one can construct a flow for which Bing's pseudo-arc [36] is the global attractor, and this set is not simply connected. Langa and Robinson [37] showed that invariant sets that enjoy a certain property related to normal hyperbolicity will be simply connected (and connected in other senses too), but such a condition is very strong and would be very hard to check in examples.

Open Question 5. Are there natural conditions under which the global attractor is path connected?

Other topological properties of global attractors are known, for example, a global attractor of a semiflow on a linear space has "trivial shape," see Garay [38] or Robinson and Sánchez-Gabites [39].

4.2.2. An Analytic Characterisation of the Global Attractor. A "complete" orbit $u(\cdot)$ is a solution of the PDE (or ODE) which is defined for all $t \in \mathbb{R}$. In general we do not expect the solutions of a PDE to lie on a complete orbit, since we cannot define $S(t)$ for $t<0$. We say that a complete orbit $u: \mathbb{R} \rightarrow H$ is bounded if there is some $M$ such that $|u(t)| \leq M$ for all $t \in \mathbb{R}$.

The global attractor consists of all bounded complete orbits. This is a noteworthy result, since it gives an analytic characterisation of the global attractor as the set of a particular class of solutions, even though our original definition was a dynamical one.

Theorem 12. The global attractor $\mathscr{A}$ is the union of all the complete bounded orbits.

Proof. Let $\mathcal{O}$ be a complete bounded orbit, and assume that $\mathcal{O}$ is not contained in $\mathscr{A}$; then for some $\epsilon>0$ there is a point $x \in \mathcal{O}$ with $x \notin N(\mathscr{A}, \epsilon)$. However, since $\mathscr{A}$ attracts bounded sets, for $t$ large enough

$$
\operatorname{dist}(S(t) z, \mathscr{A})<\epsilon \quad \forall z \in \mathcal{O} \text {. }
$$

Since $\mathcal{O}$ is a complete orbit, $x=S(t) \tilde{x}$ for some $\tilde{x} \in \mathcal{O}$; (82) now gives a contradiction and shows that all complete bounded orbits lie in $\mathscr{A}$.

Now take $x \in \mathscr{A}$; for $t>0$ it is immediate that $x(t):=$ $S(t) x$ is bounded since it lies in $\mathscr{A}$ by invariance of $\mathscr{A}$. We now have to construct $x(t)$ for $t<0$. Since $\mathscr{A}$ is invariant, there exists an $x_{-1} \in \mathscr{A}$ such that $S(1) x_{-1}=x$ (this $x_{-1}$ need not be unique unless $S(1)$ is injective, see Theorem 13, below). Let $x(-1+t)=S(t) x_{-1}$ for $t \in[0,1)$. Now find $x_{-2} \in \mathscr{A}$ such that $S(1) x_{-2}=x_{-1}$, and set $x(-2+t)=S(t) x_{-2}$ for $t \in[0,1)$. Continue inductively in this way to define $x(t)$ for all $t<0$. That $x(\cdot)$ is a trajectory follows from the continuity of $S(\cdot)$ and the semigroup property.

In this way we have shown that every $x \in \mathscr{A}$ lies on a complete bounded orbit, and, hence, that $\mathscr{A}$ is precisely the union of all such orbits.

It is interesting to note that in certain situations there are complete orbits that are not bounded. For example, Constantin et al. [40] explore the sets of solutions of the 2D Navier-Stokes equations (with periodic boundary conditions) that exist for all time and grow at the same rate as solutions of the linear Stokes problem as $t \rightarrow-\infty$.

4.2.3. A Dynamical System on the Attractor. If the semigroup $S(t)$ is injective on $\mathscr{A}$ (in the sense of Section 2.5) then the dynamics, restricted to $\mathscr{A}$, actually define a dynamical system; that is, $\left.S(t)\right|_{\mathscr{A}}$ makes sense for all $t \in \mathbb{R}$, not just for $t \geq 0$. This is one good reason for investigating the dynamics on the attractor. The importance of this result is emphasised in Hale [31]. 
Theorem 13. If the semigroup is injective on $\mathscr{A}$ then every trajectory on $\mathscr{A}$ is defined for all $t \in \mathbb{R}$, and (67) holds for all $t \in \mathbb{R}$. In particular,

$$
\left(\mathscr{A},\{S(t)\}_{t \in \mathbb{R}}\right)
$$

is a dynamical system.

Proof. For each $u \in \mathscr{A}$ we know that $u \in S(t) \mathscr{A}$, and so there exists a unique $v \in \mathscr{A}$ with $S(t) v=u$. We define $S(-t) u=v$ to give $S(t)$ for all $t \in \mathbb{R}$ and, hence, obtain (67) for $t<0$ also. Since $\mathscr{A}$ is compact, it follows that $S(-t)$ as defined here is continuous on $\mathscr{A}$. Thus $S(t)$ is a continuous map from $\mathscr{A}$ into itself for all $t \in \mathbb{R}$, and it is easy to check that $S(t) S(s)=S(t+s)$ for all $t, s \in \mathbb{R}$.

4.2.4. Unstable Manifolds in the Attractor. To investigate the structure of the attractor further, we need to recall the definition of unstable manifolds.

Definition 14. The unstable manifold of an invariant set $X$ is the set

$W^{u}(X)$

$=\left\{u_{0}:\right.$ there is a complete orbit $u: \mathbb{R} \longrightarrow H$

with $u(0)=u_{0}$ and $\operatorname{dist}\left(S(-t) u_{0}, X\right) \longrightarrow 0$ as $\left.t \longrightarrow \infty\right\}$.

Now, the unstable manifold of any invariant set (in particular of any fixed point or periodic orbit) is contained in the attractor.

Theorem 15. If $X$ is a compact invariant set, then

$$
W^{u}(X) \subset \mathscr{A} \text {. }
$$

Proof. Let $u \in W^{u}(X)$; then by definition (Definition 14) $u$ lies on the complete orbit $Y=\cup_{t \in \mathbb{R}} u(t)$. As $t \rightarrow-\infty$ we know that $\operatorname{dist}(u(t), X) \rightarrow 0$, and as $t \rightarrow \infty$ we know that $\operatorname{dist}(u(t), \mathscr{A}) \rightarrow 0$, so the orbit $u(t)$ is bounded. Thus $u$ lies on a complete bounded orbit, and by Theorem $12, u \in \mathscr{A}$.

\section{Asymptotic Bounds on Solutions}

Central to proving results on existence of an attractor for the Navier-Stokes equations (and for other PDEs) are various bounds on the norms of solutions. In order to prove the existence of solutions for all time, we have to prove that some norm of the solution is bounded for all time. Because of the strong dissipation in many parabolic problems, it is often a short step from these bounds to time-asymptotic bounds that are independent of the initial conditions, and these are essentially what we require for the existence of an attractor.

We will give our estimates in terms of the dimensionless Grashof number which measures the relative strength of the forcing and viscosity and is defined as

$$
G=\frac{|f|}{v^{2} \lambda_{1}}
$$

(recall that we use $|f|$ to denote the $L^{2}$ norm of $f$ ). For an alternative definition that uses the norm of $f$ in $V^{*}$ rather than in $L^{2}$ see Robinson [41] and Section 6.4.

Although the Grashof number is mathematically convenient (it only makes use of terms that occur explicitly in the equation), it is more conventional to discuss qualitative properties of fluid flows in terms of the Reynolds Number, $\operatorname{Re}=U \ell / \nu$, where $U$ is a temporal and spatial average of the velocity $\left(U^{2}=L^{-2}\left\langle\|u\|_{L^{2}}^{2}\right\rangle\right)$ and $\ell$ is the forcing scale. This issue is discussed in detail by Gibbon and Pavliotis [42]; making use of an analysis due to Doering and Foias [43] they show that when $G$ is large

$$
G \leq c\left(\operatorname{Re}^{2}+\mathrm{Re}\right)
$$

for an appropriate definition of the Reynolds number Re.

5.1. A Compact Absorbing Set When $f \in H$. Our aim here is to show that when $f \in H$ and $u_{0} \in H$ there is a bounded set in $V$ that is absorbing. Since $H^{1}$ is compactly embedded in $L^{2}$, this yields a compact absorbing set in $L^{2}$. We obtain such a set in two stages. First we show that there is a bounded absorbing set in $H$ and then use this (and an auxiliary estimate) to prove the existence of an absorbing set in $V$.

Although the existence of an absorbing set in $V$ for the 2D equations was first shown (in different terminology) by Foias and Prodi [44], the proof of the existence of a global attractor for the 2D Navier-Stokes equations was first published by Ladyzhenskaya in 1972 (see [45], for an English translation) and later, along with many other important results, by Foias and Temam [46].

5.1.1. A bounded Absorbing Set in $H$. To prove the existence of an absorbing set we will need the following simple lemma. To prove this one simply multiplies by the integrating factor $e^{a t}$ and integrates.

Lemma 16 (Gronwall). If $X \in L^{1}(0, T)$ is positive almost everywhere and

$$
\frac{\mathrm{d} X}{\mathrm{~d} t} \leq a X+b
$$

then

$$
X(t) \leq X(0) e^{a t}+e^{a t}(b-X(0)) \quad \forall t \in[0, T] .
$$

We now prove the existence of an absorbing set in $H$ and an asymptotic bound on the integral (in time) of the norm in $V$.

Proposition 17. Given $f \in H$ let

$$
\varrho_{0}^{2}:=\frac{|f|^{2}}{v^{2} \lambda_{1}^{2}}=v^{2} G^{2} .
$$

Then for any $\rho_{0}>\varrho_{0}$ and $u_{0} \in H$ there exists a time $t_{\rho_{0}}\left(\left|u_{0}\right|\right)$ such that

$$
\left|S(t) u_{0}\right| \leq \rho_{0} \quad \forall t \geq t_{\rho_{0}}\left(\left|u_{0}\right|\right)
$$


that is, the ball in $L^{2}$ of radius $\rho_{0}$ is absorbing. One also has

$$
\nu \int_{t}^{t+1}|D u(s)|^{2} \mathrm{~d} s \leq I_{1}:=\rho_{0}^{2}+\nu^{3} \lambda_{1} G^{2} \quad \forall t \geq t_{\rho_{0}}\left(\left|u_{0}\right|\right)
$$

$$
\limsup _{T \rightarrow \infty} \frac{1}{T} \int_{0}^{T}|D u(t)|^{2} \mathrm{~d} t \leq \frac{|f|^{2}}{v^{2} \lambda_{1}}=v^{2} \lambda_{1} G^{2} .
$$

Proof. We take the inner product of

$$
\frac{\mathrm{d} u}{\mathrm{~d} t}+v A u+B(u, u)=f
$$

with $u$ to obtain

$$
\frac{1}{2} \frac{\mathrm{d}}{\mathrm{d} t}|u|^{2}+\nu|D u|^{2}+(B(u, u), u)=(f, u) .
$$

Since $(B(u, u), u)=0$ this gives

$$
\frac{1}{2} \frac{\mathrm{d}}{\mathrm{d} t}|u|^{2}+\nu|D u|^{2} \leq|f||u| .
$$

We now use the Poincaré inequality on the $|D u|$ term

$$
|D u|^{2} \geq \lambda_{1}|u|^{2}
$$

and Young's inequality on the right-hand side to write

$$
\frac{1}{2} \frac{\mathrm{d}}{\mathrm{d} t}|u|^{2}+v \lambda_{1}|u|^{2} \leq \frac{v \lambda_{1}}{2}|u|^{2}+\frac{1}{2 \nu \lambda_{1}}|f|^{2} .
$$

Tidying this up gives

$$
\frac{\mathrm{d}}{\mathrm{d} t}|u|^{2} \leq-v \lambda_{1}|u|^{2}+\frac{1}{\nu \lambda_{1}}|f|^{2},
$$

and then from Gronwall's inequality (Lemma 16)

$$
|u(t)|^{2} \leq\left|u_{0}\right|^{2} e^{-\nu \lambda_{1} t}+\frac{|f|^{2}}{\nu^{2} \lambda_{1}^{2}}\left(1-e^{-\nu \lambda_{1} t}\right) .
$$

From this it is clear that given any $\rho_{0}>\varrho_{0}$ there exists a time $t_{0}$, which depends only on $\rho_{0}$ and $\left|u_{0}\right|$ such that

$$
|u(t)|^{2} \leq \rho_{0}^{2} \quad \forall t \geq t_{\rho_{0}}\left(\left|u_{0}\right|\right) .
$$

If we return to (96) and use the Poincaré inequality on the $|u|$ term on the right-hand side we can then apply Young's inequality to obtain

$$
\frac{\mathrm{d}}{\mathrm{d} t}|u(t)|^{2}+\nu|D u|^{2} \leq \frac{|f|^{2}}{\nu \lambda_{1}},
$$

and integrating from $t$ to $t+1$ yields

$$
v \int_{t}^{t+1}|D u(s)|^{2} \mathrm{~d} s \leq|u(t)|^{2}+\frac{|f|^{2}}{v \lambda_{1}},
$$

which implies that for $t \geq t_{\rho_{0}}\left(\left|u_{0}\right|\right)$

$$
\nu \int_{t}^{t+1}|D u(s)|^{2} \mathrm{~d} s \leq \rho_{0}^{2}+\nu^{3} \lambda_{1} G^{2},
$$

yielding (107). A similar integration yields (93).

(Note that one can obtain a similar result with the weaker assumption that $f \in V^{*}$, by replacing the right-hand side of (96) by $\|f\|_{*}|D u|$ and continuing similarly.)
5.1.2. A Bounded Absorbing Set in V. The existence of an absorbing set in $V$ for the 2D equations was first shown (in a different terminology) by Foias and Prodi [44]. This is the crucial ingredient for proving the existence of a global attractor. Although in the proof we use the orthogonality property $(B(u, u), A u)=0$, which is only valid for periodic boundary conditions in $2 \mathrm{D}$, the same result (with a slightly more involved argument and weaker estimates) holds for Dirichlet boundary conditions.

Proposition 18. Given $f \in H$ and $\rho_{0}>\varrho_{0}$ let

$$
\rho_{1}^{2}:=\nu^{3} \lambda_{1}^{2} G^{2}+\rho_{0}^{2} \nu+\nu^{2} \lambda_{1} G^{2} .
$$

Then for any $u_{0} \in H$

$$
\left\|S(t) u_{0}\right\|_{V} \leq \rho_{1} \quad \forall t \geq t_{\rho_{0}}\left(\left|u_{0}\right|\right)+1,
$$

where $t_{\rho_{0}}(\cdot)$ is the same as in Proposition 17; that is, the ball in $H^{1}$ of radius $\rho_{1}$ is absorbing. One also has

$$
\begin{gathered}
v \int_{t}^{t+1}|D u(s)|^{2} d s \leq \rho_{1}^{2}+v^{3} \lambda_{1} G^{2} \quad \forall t \geq t_{\rho_{0}}\left(\left|u_{0}\right|\right) \\
\limsup _{T \rightarrow \infty} \frac{1}{T} \int_{t}^{t+T}|A u(s)|^{2} d s \leq \frac{|f|^{2}}{v^{2}}=\lambda_{1}^{2} v^{2} G^{2}
\end{gathered}
$$

Note that by an appropriate choice of $\rho_{0}$ we can take any $\rho_{1}>\varrho_{1}$ where

$$
\varrho_{1}=\left[1+\nu \lambda_{1}+\nu^{2} \lambda_{1}^{2}\right] \nu G^{2} .
$$

Proof. To prove the existence of this absorbing set we use a "trick" - a double integration in time-which can be formalised as the "uniform Gronwall lemma" (see Lemma 1.1 in Chapter III of Temam [18], e.g., although the statement of this as a formal lemma somewhat obscures the underlying idea). We take the inner product of (18) with $A u$ to give

$$
\frac{1}{2} \frac{\mathrm{d}}{\mathrm{d} t}|D u|^{2}+v|A u|^{2}+B(u, u, A u)=(f, A u) .
$$

We now use an orthogonality property $(B(u, u), A u)=0$ and the Cauchy-Schwarz inequality to rewrite this as

$$
\frac{1}{2} \frac{\mathrm{d}}{\mathrm{d} t}|D u|^{2}+\nu|A u|^{2} \leq \frac{|f|^{2}}{2 \nu}+\frac{v}{2}|A u|^{2} .
$$

Dropping the $|A u|^{2}$ terms we have

$$
\frac{\mathrm{d}}{\mathrm{d} t}|D u|^{2} \leq \frac{|f|^{2}}{v} .
$$

We now use the double integration trick. First integrate this equation between $s$ and $t+1$, with $t \leq s<t+1$, which gives

$$
|D u(t+1)|^{2} \leq \frac{|f|^{2}}{v}+|D u(s)|^{2}
$$


( since $0<t+1-s \leq 1$ ). We now integrate both sides with respect to $s$ between $t-1$ and $t$ and obtain

$$
|D u(t+1)|^{2} \leq \frac{|f|^{2}}{v}+\int_{t}^{t+1}|D u(s)|^{2} \mathrm{~d} s .
$$

Now provided that $t \geq t_{\rho_{0}}\left(\left|u_{0}\right|\right)$ we can use (107) to give

$$
|D u(t+1)|^{2} \leq \frac{|f|^{2}}{v}+\frac{1}{v}\left(\rho_{0}^{2}+v^{3} \lambda_{1} G^{2}\right) .
$$

We note also that if we return to (111) then we have

$$
\frac{\mathrm{d}}{\mathrm{d} t}|D u|^{2}+v|A u|^{2} \leq \frac{|f|^{2}}{\nu}
$$

and integrating from $t$ to $t+T$ we obtain

$$
|D u(t+T)|^{2}+v \int_{t}^{t+T}|A u(s)|^{2} \mathrm{~d} s \leq|D u(t)|^{2}+\frac{T|f|^{2}}{v},
$$

which yields (107) and (108).

5.2. Smoothness When $f \in H^{s}$. In fact with higher regularity of $f$ we can obtain much better bounds on the functions in the attractor. We will use the following estimates on the nonlinear term, which follow easily from the fact that $H^{s}$ is an algebra for $s>1$. We use $\|\cdot\|_{s}$ to denote the norm in $H^{s}$.

Lemma 19. For any $s>1$

$$
\left|\left(B(u, u), A^{s} u\right)\right|_{L^{2}} \leq c\|u\|_{s}^{2}\|u\|_{s+1} \quad \forall u \in H^{s+1} .
$$

We use this to prove better asymptotic regularity of solutions when $f$ is more regular.

Corollary 20. If $f \in H^{s}$ then $\mathscr{A}$ is bounded in $H^{s+1}$.

Proof. Suppose as an inductive hypothesis that for any $u_{0} \in$ $\mathscr{A}$,

$$
\left\|u_{0}\right\|_{k}^{2} \leq R_{k}, \quad \int_{0}^{1}\left\|S(t) u_{0}\right\|_{k+1}^{2} \mathrm{~d} t \leq I_{k+1} .
$$

Note that this holds for $k=1$ since the attractor in bounded in $H^{1}$; the integral bound follows from (117). We show that while $k \leq s$ it follows that (119) holds with $k$ replaced by $k+1$.

Now, for any $u \in \mathscr{A}$, we have $u=S(1) u_{0}$ for some $u_{0} \in \mathscr{A}$, since $\mathscr{A}$ is invariant. It follows from (119) that there exists a $t_{0} \in[0,1]$ such that

$$
\left\|S(t) u_{0}\right\|_{k+1}^{2} \leq I_{k+1} .
$$

We now consider the solution starting at $u_{0}$, noting that $u=$ $S\left(1-t_{0}\right) u_{0}$.

Take the inner product of (18) with $A^{k+1} u$ to obtain

$$
\begin{aligned}
& \frac{1}{2} \frac{\mathrm{d}}{\mathrm{d} t}\|u\|_{k+1}^{2}+v\|u\|_{k+2}^{2} \\
& \leq\left|\left(B(u, u), A^{k+1} u\right)\right|+\|f\|_{k-1}\|u\|_{k+2} \\
& \leq c_{k}\|u\|_{k+1}^{2}\|u\|_{k+2}+\|f\|_{k-1}\|u\|_{k+2},
\end{aligned}
$$

using Lemma 19. After using Young's inequality on the righthand side this becomes

$$
\frac{\mathrm{d}}{\mathrm{d} t}\|u\|_{k+1}^{2}+v\|u\|_{k+2}^{2} \leq \frac{c_{k}^{2}}{v}\|u\|_{k+1}^{4}+\frac{1}{v}\|f\|_{k-1}^{2} .
$$

If we drop the second term on the LHS and use Gronwall's inequality we can deduce that

$$
\begin{aligned}
\|u\|_{k+1}^{2} & =\left\|u\left(1-t_{0}\right)\right\|_{k+1}^{2} \\
& \leq\left(\left\|u_{0}\right\|_{k+1}^{2}+\frac{\|f\|_{k-1}^{2}}{2 v}\right) \exp \left(\frac{c_{k}^{2}}{v} \int_{0}^{1-t_{0}}\|u(s)\|_{k+1}^{2} \mathrm{~d} s\right) \\
& \leq\left(R_{k}+\frac{\|f\|_{k-1}^{2}}{2 v}\right) \exp \left(\frac{c_{k}^{2} I_{k+1}}{v}\right)=: R_{k+1} .
\end{aligned}
$$

Now return to (122) and integrate from 0 to 1 , starting at $u(0)=u$, to obtain

$$
\begin{aligned}
& \frac{1}{2}\left\|S(1) u_{0}\right\|^{2}+v \int_{0}^{1}\|S(t) u\|_{k+1}^{2} \mathrm{~d} t \\
& \leq \frac{c_{k}^{2} R_{k+1}^{2}}{v}+\frac{1}{v}\|f\|_{k-1}^{2}=: I_{k+2} .
\end{aligned}
$$

Open Question 7. The previous bounds are very crude. Can one find the optimal dependence of the $H^{k}$ bounds on the attractor in terms of norms of $f$ ?

For one approach to this, based on the time analyticity of solutions, see Foias et al. [47]. $k \in \mathbb{N}$

We say that a function $f$ is smooth if $f \in H^{k}$ for every

Corollary 21. If $f$ is smooth then the Navier-Stokes attractor consists of smooth functions.

To close this section we note that it is in fact possible to obtain asymptotic bounds on $|A u|$ when $f \in H$ (the previous proof requires $f \in V$ ), by making estimates on the time derivative of the equation $[16,48]$. We will use the existence of an absorbing set in $D(A) \subset H^{2}$ in Sections 8 and 9. For another approach to higher regularity see Guillopé [49] or Temam [18].

5.3. Gevrey Regularity. We now use the theory of Gevrey regularity, developed by Foias and Temam [50], to show that if $f$ is real analytic then the functions on the attractor are all real analytic, in a uniform way.

A function $f(x)$ is real analytic, that is, it can be represented locally by its Taylor series expansion, if and only if its derivatives satisfy

$$
\sup _{x}\left|D^{\beta} f(x)\right| \leq M|\beta| ! \tau^{-|\beta|}
$$


for some $M$ and $\tau$ see John [51], for example. This motivates the definition of the analytic Gevrey class $D\left(e^{\tau A^{1 / 2}}\right)$; this consists of functions such that

$$
\left|e^{\tau A^{1 / 2}} u\right|<+\infty,
$$

where $e^{\tau^{1 / 2} A}$ is defined using the power series for exponentials

$$
e^{\tau A^{1 / 2}}=\sum_{n=0}^{\infty} \frac{\tau^{n}}{n !} A^{n / 2}
$$

Writing $u$ as a Fourier expansion

$$
u=\sum_{j \in \mathbb{Z}^{2}} u_{j} e^{i j \cdot x}
$$

we have

$$
\left|e^{\tau A^{1 / 2}} u\right|^{2}=\sum_{j \in \mathbb{Z}^{2}} e^{2 \tau|j|}\left|u_{j}\right|^{2}
$$

In particular, therefore, if $u \in D\left(e^{\tau A^{1 / 2}}\right)$ the Fourier coefficients of $u$ must decay exponentially fast.

Foias and Temam [50] (see also Doering and Gibbon [2] for an alternative proof) showed that if $f \in D\left(e^{\sigma A^{1 / 2}}\right)$ for some $\sigma>0$ then $u(t)$ is bounded in $D\left(A^{1 / 2} e^{\tau A^{1 / 2}}\right)$, and

$$
\left|A^{1 / 2} e^{\tau A^{1 / 2}} u\right| \leq K \quad \forall t \geq T,
$$

$T$ and $K$ depend only on $|D u(0)|$.

We give the proof here, following Foias and Temam's paper closely. We assume the following inequality, which is Lemma 2.1 in Foias and Temam [50]; if $u \in D\left(A e^{\tau A^{1 / 2}}\right)$ for some $\tau>0$ then

$$
\left|\left(e^{\tau A^{1 / 2}} B(u, u), e^{\tau A^{1 / 2}} A u\right)\right| \leq c\left|e^{\tau A^{1 / 2}} A^{1 / 2} u\right|^{3 / 2}\left|e^{\tau A^{1 / 2}} A u\right|^{3 / 2}
$$

for some $c>0$. In order to make the notation more compact, we can write

$$
(u, v)_{\tau}=\left(e^{\tau A^{1 / 2}} u, e^{\tau A^{1 / 2}} v\right)
$$

and $|u|_{\tau}=\left|e^{\tau A^{1 / 2}} u\right|$. The previous inequality is now

$$
\left|(B(u, u), A u)_{\tau}\right| \leq c\left\|A^{1 / 2} u\right\|_{\tau}^{3 / 2}\|A u\|_{\tau}^{3 / 2}
$$

Theorem 22. If $f \in D\left(e^{\sigma A^{1 / 2}}\right)$ then for $t \leq T\left(|f|_{\sigma}+\left|A^{1 / 2} u(0)\right|\right)$ one has

$$
\left|A^{1 / 2} e^{\phi(t) A^{1 / 2}} u(t)\right| \leq K\left(\|f\|_{\sigma},\left|A^{1 / 2} u(0)\right|\right) \quad \forall 0 \leq t \leq T,
$$

where $\phi(t)=\min (\sigma, t)$.
Proof. Taking the scalar product with $A u$ in $D\left(e^{\tau A^{1 / 2}}\right)$ leads to an equation for $y=\left\|A^{1 / 2} u\right\|_{\tau}$ like $\dot{y} \leq K y^{3}$. Not only do the solutions of this equation blow up in a finite time, but also we need to control $\left\|A^{1 / 2} u(0)\right\|_{\tau}$ in order to control $\left\|A^{1 / 2} u(t)\right\|_{\tau}$; we would need to start with analyticity in order to prove it.

The trick to get round this is to define $\phi(t)=\min (t, \sigma)$, and take the scalar product of

$$
\frac{\mathrm{d} u}{\mathrm{~d} t}+v A u+B(u, u)=f
$$

with $e^{2 \phi(t) A^{1 / 2}} A u$ to obtain

$$
\begin{aligned}
& \left(\frac{\mathrm{d} u}{\mathrm{~d} t}, e^{2 \phi(t) A^{1 / 2}} A u\right)+v\left|e^{\phi(t) A^{1 / 2}} A u\right|^{2} \\
& =\left(e^{\phi(t) A^{1 / 2}} f, e^{\phi(t) A^{1 / 2}} A u\right)-\left(e^{\phi(t) A^{1 / 2}} B(u, u), e^{\phi(t) A^{1 / 2}} A u\right) \\
& =(f, A u)_{\phi}-(B(u, u), A u)_{\phi} \\
& \leq\|f\|_{\phi}\|A u\|_{\phi}+c\left\|A^{1 / 2} u\right\|_{\phi}^{3 / 2}\|A u\|_{\phi}^{3 / 2} \\
& \leq \frac{v}{4}\|A u\|_{\phi}^{2}+\frac{2}{v}\|f\|_{\phi}^{2}+\frac{c}{v^{3}}\left\|A^{1 / 2} u\right\|_{\phi}^{6} .
\end{aligned}
$$

The left-hand side of the equation can be bound as

$$
\begin{aligned}
& \left(e^{\phi(t) A^{1 / 2}} \frac{\mathrm{d} u}{\mathrm{~d} t}, e^{\phi(t) A^{1 / 2}} A u\right) \\
& =\left(A^{1 / 2} \frac{\mathrm{d}}{\mathrm{d} t}\left(e^{\phi(t) A^{1 / 2}} u(t)\right)\right. \\
& \left.\quad-\frac{\mathrm{d} \phi}{\mathrm{d} t} A e^{\phi(t) A^{1 / 2}} u(t), e^{\phi(t) A^{1 / 2}} A^{1 / 2} u(t)\right) \\
& =\frac{1}{2} \frac{\mathrm{d}}{\mathrm{d} t}\left|A^{1 / 2} e^{\phi(t) A^{1 / 2}} u(t)\right|^{2} \\
& =\frac{1}{2} \frac{\mathrm{d}}{\mathrm{d} t}\left\|A^{1 / 2} u\right\|_{\phi}^{2}-\frac{\mathrm{d} \phi}{\mathrm{d} t}\left(A u, A^{1 / 2} u\right)_{\phi} \\
& \geq \frac{1}{2} \frac{\mathrm{d}}{\mathrm{d} t}\left\|A^{1 / 2} u\right\|_{\phi}^{2}-\|A u\|_{\phi(t)}\left\|A^{1 / 2} u\right\|_{\phi} \\
& \geq \frac{1}{2} \frac{\mathrm{d}}{\mathrm{d} t}\left\|A^{1 / 2} u\right\|_{\phi}^{2}-\frac{v}{4}\|A u\|_{\phi}^{2}-\frac{1}{v}\left\|A^{1 / 2} u\right\|_{\phi}^{2} .
\end{aligned}
$$

We therefore have

$$
\begin{aligned}
& \frac{\mathrm{d}}{\mathrm{d} t}\left\|A^{1 / 2} u\right\|_{\phi}+v\|A u\|_{\phi}^{2} \\
& \leq \frac{4}{v}\|f\|_{\phi}^{2}+\frac{2}{v}\left\|A^{1 / 2} u\right\|_{\phi}^{2}+\frac{c}{v^{3}}\left\|A^{1 / 2} u\right\|_{\phi}^{6} \\
& \leq \frac{4}{v}\|f\|_{\phi}^{2}+c+\frac{c}{v^{3}}\left\|A^{1 / 2} u\right\|_{\phi}^{6}
\end{aligned}
$$


Now we can set

$$
y(t)=1+\left\|A^{1 / 2} u(t)\right\|_{\phi(t)}^{2}
$$

and we have

$$
\frac{\mathrm{d} y}{\mathrm{~d} t} \leq K y^{3}
$$

with

$$
K=\frac{4}{\nu}\|f\|_{\sigma}^{2}+c+\frac{c}{\nu}
$$

The solution of (140) is

$$
y(t) \leq \frac{1}{y(0)^{-2}-2 K t},
$$

and so $y(t) \leq 2 y(0)$ for $t \leq\left(4 K y(0)^{2}\right)^{-1}$. Since $\phi(0)=0$, we have

$$
y(0)=1+\left\|A^{1 / 2} u(0)\right\|^{2},
$$

and so for $t \leq T\left(\left|A^{1 / 2} u(0)\right|,\|f\|_{\sigma}\right)$, we have

$$
\left\|A^{1 / 2} u(t)\right\|_{\phi} \leq K\left(\left|A^{1 / 2} u(0)\right|,\|f\|_{\sigma}\right),
$$

and the theorem follows.

The following is an immediate corollary of Theorem 22 .

Corollary 23. Suppose that $f \in D\left(e^{\tau A^{1 / 2}}\right)$ for some $\tau>0$. Then there exists $a>0$ and a constant $\varrho_{G}$ that depends only on $\|f\|_{\tau}$ such that

$$
\left|A^{1 / 2} e^{\sigma A^{1 / 2}} u\right| \leq \varrho_{G} \quad \forall u \in \mathscr{A}
$$

Proof. Let $T=T\left(\varrho_{1},\|f\|_{\tau}\right)$ from Theorem 22. Take $u \in \mathscr{A}$. Then $u=S(T) u_{0}$ for some $u_{0} \in \mathscr{A}$. Since $u_{0} \in \mathscr{A},\left|D u_{0}\right| \leq \varrho_{1}$, and so we obtain

$$
\left\|A^{1 / 2} u\right\|_{\phi(T)}=\left\|A^{1 / 2} u(T)\right\|_{\phi(T)} \leq K\left(\varrho_{1},\|f\|_{\tau}\right)
$$

uniformly over $\mathscr{A}$, using (144).

The $\sigma$ in the exponent reflects the size of the radius of analyticity of $u$. Some interesting work on obtaining the maximum radius of analyticity can be found in Kukavica [52].

We will use the real analyticity of solutions on the attractor in Section 8.2.2 to show that elements of the attractor can be distinguished by their values at a finite number of points in $\Omega$.

Open Question 8. If $f \in D\left(e^{\tau A^{1 / 2}}\right)$ then one can show (cf. (125)) that $\|f\|_{k} \leq \tau^{-k} k !\|u\|_{\tau}$ see Friz and Robinson [53], for example. Can one obtain sufficiently good bounds in answer to Open Question 7 to deduce Gevrey regularity for the attractor given these bounds on $\|f\|_{k}$ ?

\section{Finite-Dimensional Attractors}

We now show that the attractor of the 2D Navier-Stokes equations is a finite-dimensional subset of the infinite-dimensional phase space $H$. This was first shown by Ladzyhenskaya [54].

6.1. The (Upper) Box-Counting Dimension. The box-counting dimension, which we will write as $d_{\text {box }}(X)$, is based on counting the number of closed balls of a fixed radius $\epsilon$ needed to cover $X$.

We denote the minimum number of balls in such a cover by $N(X, \epsilon)$. If $X$ were a line, we would expect that $N(X, \epsilon) \sim$ $\epsilon^{-1}$, if $X$ were a surface, we would have $N(X, \epsilon) \sim \epsilon^{-2}$, and for a (3-) volume, we would have $N(X, \epsilon) \sim \epsilon^{-3}$. So one possible method for obtaining a general measure of dimension would be to say that $X$ has dimension $d$ if $N(X, \epsilon) \sim \epsilon^{-d}$. Accordingly, we make the following definition.

Definition 24. The box-counting dimension of $X, d_{\text {box }}(X)$, is given by

$$
d_{\text {box }}(X)=\limsup _{\epsilon \rightarrow 0} \frac{\log N(X, \epsilon)}{\log (1 / \epsilon)},
$$

where we allow the limit in (147) to take the value $+\infty$.

Note that it follows from the definition that if $d>$ $d_{\text {box }}(X)$, then for sufficiently small $\epsilon$,

$$
N(X, \epsilon) \leq \epsilon^{-d}
$$

For further discussion of the box-counting dimension see Eden et al. [55], Falconer [56, 57], or Robinson [16, 58].

6.2. Dimension Estimates. Techniques to show that invariant sets have finite box-counting dimension date back to MalletParet [59]. His argument, valid for invariant subsets of Hilbert spaces, admits generalisation to Banach spaces (see [60], recently updated in [61]). However, the method that gives the best estimates is restricted to Hilbert spaces and was developed in infinite-dimensional spaces by Constantin and Foias [62] and Constantin et al. [7] [see also [63] or [18]], after the finite-dimensional approach of Douady and Oesterlé [64].

The idea is to study the evolution of infinitesimal $n$ dimensional volumes as they evolve under the flow and try to find the smallest dimension $n$ at which we can guarantee that all such $n$-volumes contract asymptotically. We will not give the analysis in detail but merely in outline.

We will consider an abstract problem, written as

$$
\frac{\mathrm{d} u}{\mathrm{~d} t}=F(u(t)) \quad u(0)=u_{0}
$$

with $u_{0}$ contained in a Hilbert space $H$, whose norm we denote by $|\cdot|$. We assume that the equation has unique solutions given by $u\left(t ; u_{0}\right)=S(t) u_{0}$ and a compact global attractor $\mathscr{A}$.

We want to start off with an orthogonal set of infinitesimal displacements near an initial point $u_{0} \in \mathscr{A}$ and then watch how the volume they form evolves under the flow. 
To study the evolution of this volume we have to study the evolution of a set of infinitesimal displacements $\delta x^{(i)}(t)$ about the trajectory $u(t)$. We suppose that the evolution of these displacements is given by the linearised equation

$$
\frac{\mathrm{d} U}{\mathrm{~d} t}=F^{\prime}(u(t)) U(t) \quad U(0)=\xi,
$$

which we write as

$$
\frac{\mathrm{d} U}{\mathrm{~d} t}=L\left(t ; u_{0}\right) U(t) \quad U(0)=\xi .
$$

The validity of such a linearisation is one of the main points to check when applying this theory rigorously. To this end we make the following definition.

Definition 25. We say that $S(t)$ is uniformly differentiable on $\mathscr{A}$ if for every $u \in \mathscr{A}$ there exists a linear operator $\Lambda(t, u)$ : $H \rightarrow H$, such that, for all $t \geq 0$,

$$
\begin{aligned}
\sup _{u, v \in \mathscr{A} ; 0<|u-v| \leq \epsilon} \frac{|S(t) v-S(t) u-\Lambda(t, u)(v-u)|}{|v-u|} & \longrightarrow 0 \\
\text { as } \epsilon & \longrightarrow 0 \\
\sup _{u \in \mathscr{A}}\|\Lambda(t, u)\|_{\text {op }}<\infty \quad \text { for each } t \geq 0 . &
\end{aligned}
$$

Although this is straightforward to check for ordinary differential equations, its proof in the PDE context will often involve technical difficulties.

Heuristically speaking the growth rate of each infinitesimal displacement $\delta x^{(j)}$ will be related to the eigenvalues of $L$. In particular, the length of an infinitesimal displacement in the $\lambda$-eigendirection is $e^{\lambda t} \delta(0)$ at time $t$; the growth rate is $\lambda$, associated with the eigendirection of $L$ with eigenvalue $\lambda$. The size of a small 2-volume with sides in two different eigendirections would be

$$
e^{\left(\lambda_{1}+\lambda_{2}\right) t}
$$

and so the growth "rate" is $\lambda_{1}+\lambda_{2}$. The growth rate of an $n$ volume made of infinitesimal directions in $n$ eigendirections would be

$$
\lambda_{1}+\cdots+\lambda_{n}
$$

If we can make sure that this growth rate must be negative then we know that $n$-volumes contract. It is true, though by no means immediate, that if all $n$-volumes contract then the dimension of the attractor must be smaller than $n$. In the finite-dimensional setting this result is due to Douady and Oesterlé [64], while in the infinite-dimensional case it was proved by Constantin and Foias [62] and Constantin et al. [7].

To extract the "growth rate," we consider

$$
\sum_{j=1}^{n}\left(\phi_{j}, L\left(t ; u_{0}\right) \phi_{j}\right)
$$

over all possible orthonormal collections of $n$ elements $\left\{\phi_{j}\right\}_{j=1}^{n}$ of $H$. The idea is, essentially, that the maximum over all choices of $\phi_{j}$ gives the largest possible growth rate, that is, the sum of the $n$ largest eigenvalues of $L$. A more compact notation for (155) is

$$
\operatorname{Tr}\left(L\left(t ; u_{0}\right) P\right)
$$

where $\operatorname{Tr}$ denotes the trace in $H$ and $P$ is the orthogonal projection onto the space spanned by the $\left\{\phi_{j}\right\}_{j=1}^{n}$.

The following theorem is given in a form suitable for calculations.

Theorem 26. Suppose that $S(t)$ is uniformly differentiable on $\mathscr{A}$, and there exists a $t_{0}$ such that $\Lambda\left(t, u_{0}\right)$ is compact for all $t \geq t_{0}$. Let

$$
\widetilde{q}_{j}:=\limsup _{T \rightarrow \infty} \sup _{u_{0} \in \mathscr{A}} \sup _{\left\{\phi_{j}\right\}_{j=1}^{n}} \frac{1}{T} \int_{0}^{T} \sum_{j=1}^{n}\left(\phi_{j}, L(u(t)) \phi_{j}\right) \mathrm{d} t,
$$

and assume that $\tilde{q}_{j} \leq q_{j}$. If $q_{n}<0$ then $d_{\mathrm{box}}(\mathscr{A})<n$.

The result of Constantin and Foias gives $d_{H}(\mathscr{A})<n$ (where $d_{H}$ denotes the Hausdorff dimension) and bounds on the box-counting dimension under stronger conditions on the $\tilde{q}_{j}$. A proof of this theorem, essentially due to Brian Hunt, is given in Appendix B in Robinson [16]; see also Chepyzhov \& Ilyin [65]. A simpler proof of the same result can be obtained under the assumption that $q_{j}$ is a concave function of $j[66]$.

Before we apply this result to give dimension bounds for the attractor of the 2D Navier-Stokes equations we will need an auxiliary lemma. For a proof see Lemma 4.21 in Carvalho et al. [63].

Lemma 27. Let $A$ be a positive unbounded linear self-adjoint operator on a Hilbert space $H$, and assume that $A$ has a compact inverse. Denote its eigenvalues by $\lambda_{j}$, ordered such that $\lambda_{j+1} \geq \lambda_{j}$. Then for any choice of $m$ orthonormal elements $\left\{\phi_{j}\right\}_{j=1}^{m}$ in $H$,

$$
\sum_{j=1}^{m}\left|A^{1 / 2} \phi_{j}\right|^{2}=\sum_{j=1}^{m}\left(A \phi_{j}, \phi_{j}\right) \geq \sum_{j=1}^{m} \lambda_{j}
$$

Note that when $A$ is the Stokes operator in $\mathbb{R}^{d}$ (on a periodic domain or a $C^{2}$ bounded domain with Dirichlet boundary conditions), one can obtain an explicit bound, since its eigenvalues satisfy

$$
c j^{2 / m} \leq \lambda_{j} \leq C j^{2 / m}
$$

and then

$$
\sum_{j=1}^{m} \lambda_{j} \geq c \sum_{j=1}^{m} j^{2 / d} \geq c n^{(2 / d)+1}=c n^{(d+2) / d} .
$$

6.3. Dimension Estimate for the Navier-Stokes Equations. In order to apply the previous theory to the Navier-Stokes 
equations we must first guarantee the differentiability of solutions. We only state the result here; a proof can be found in Robinson [16], Theorem 13.20, and Exercise 13.10.

Theorem 28. The solutions of the Navier-Stokes equations in $2 D$ satisfy (152) with $\Lambda\left(t ; u_{0}\right) \xi$ the solution of the equation

$$
\frac{\mathrm{d} U}{\mathrm{~d} t}+v A U+B(u, U)+B(U, u)=0 \quad U(0)=\xi .
$$

Furthermore, $\Lambda\left(t ; u_{0}\right)$ is compact for all $t>0$.

We can now use the trace formula to find a bound on the dimension of the attractor.

Theorem 29. The attractor for the $2 \mathrm{D}$ periodic Navier-Stokes equations is finite dimensional, with

$$
d_{\text {box }}(\mathscr{A}) \leq \frac{c}{\lambda_{1}^{1 / 2} \nu}\left\langle|D u|_{L^{2}}^{2}\right\rangle^{1 / 2} \leq c G
$$

where $\langle f\rangle$ denotes the time-average $\lim _{t \rightarrow \infty} 1 / t \int_{0}^{t} f(s) \mathrm{d} s$.

The result, due to Constantin et al. [7], is also valid as stated for Dirichlet boundary conditions.

Proof. The correct form of the linearised equation is given in (161), and so

$$
L(u) w=\nu A w-B(w, \mathrm{u})-B(u, w) .
$$

Thus the time-averaged trace $\left\langle P_{n} L(u(t))\right\rangle$ is bounded by

$$
\begin{aligned}
\left\langle P_{n} L(u)\right\rangle & =\left\langle\sum_{j=1}^{n}\left(L(u) \phi_{j}, \phi_{j}\right)\right\rangle \\
& =-\left\langle\sum_{j=1}^{n}\left(-v \Delta \phi_{j}, \phi_{j}\right)\right\rangle-\left\langle\sum_{j=1}^{n} b\left(\phi_{j}, u, \phi_{j}\right)\right\rangle .
\end{aligned}
$$

In order to control the contribution from the nonlinear term we could use the simple bound

$$
\left|\left(B\left(\phi_{j}, u\right), \phi_{j}\right)\right| \leq c\left\|\phi_{j}\right\|_{L^{4}}^{2}|D u| \leq c\left|D \phi_{j}\right||D u|
$$

(using the Ladyhenskaya inequality and the fact that $\left|\phi_{j}\right|=1$ ), but this would lead to an estimate worse than (162) by a factor of $v^{-1}$ (see [16]). A better estimate can be obtained as follows.

Note that we have

$$
\sum_{j=1}^{n} b\left(\phi_{j}, u, \phi_{j}\right)=\int_{\Omega} \sum_{j=1}^{n} \sum_{i, k=1}^{2} \phi_{j i}(x) \frac{\partial u_{k}}{\partial x_{i}}(x) \phi_{j k}(x) \mathrm{d} x,
$$

and we have for each $x \in Q$

$$
\begin{aligned}
& \left|\sum_{i, k=1}^{2} \phi_{j i}(x) \frac{\partial u_{k}}{\partial x_{i}}(x) \phi_{j k}(x)\right| \\
& \leq\left(\sum_{i=1}^{2} \sum_{j=1}^{n} \phi_{j i}(x)^{2}\right)\left(\sum_{i, k=1}^{2}\left|\frac{\partial u_{k}}{\partial x_{i}}(x)\right|^{2}\right)^{1 / 2} .
\end{aligned}
$$

It follows use the Cauchy-Schwarz inequality that

$$
\left|\sum_{j=1}^{n} b\left(\phi_{j}, u, \phi_{j}\right)\right| \leq|D u||\rho|_{L^{2}}
$$

where

$$
\rho(x)=\sum_{i=1}^{2} \sum_{j=1}^{n} \phi_{j i}(x)^{2}
$$

An inequality due to Lieb \& Thirring [67], adapted appropriately to this case (details are given in [18]), allows us to bound $|\rho|_{L^{2}}$ by

$$
|\rho|_{L^{2}}^{2} \leq c \sum_{j=1}^{n}\left|D \phi_{j}\right|^{2}
$$

It follows that

$$
P_{n} L(u) \leq-v \sum_{j=1}^{n}\left|D \phi_{j}\right|^{2}+c|D u|\left(\sum_{j=1}^{n}\left|D \phi_{j}\right|^{2}\right)^{1 / 2},
$$

and so, using the Cauchy-Schwarz inequality we obtain

$$
P_{n} L(u) \leq-\frac{v}{2} \sum_{j=1}^{n}\left|D \phi_{j}\right|^{2}+\frac{c}{v}|D u|^{2} .
$$

Now taking the time average and using Lemma 27 we obtain

$$
\left\langle P_{n} L(u)\right\rangle \leq-\frac{v}{2} n^{2}+\frac{c}{v}\langle|D u|\rangle^{2} .
$$

We therefore have $\left\langle P_{n} L(u)\right\rangle<0$ provided that $n>$ $\lambda_{1}^{-1 / 2} v^{-1}\left\langle|D u|^{2}\right\rangle^{1 / 2}$. The bound in (162) now follows from (93).

The above argument provides the best bound known for the case of Dirichlet boundary conditions. By working with the equation for $\omega=\nabla \wedge u$ and using the identity $(B(u, u), A u)=0$, Constantin et al. [68] were able to improve this for periodic boundary conditions to

$$
d_{\text {box }}(\mathscr{A}) \leq c G^{2 / 3}(1+\log G)^{1 / 3} .
$$

Modulo the logarithm this bound is known to be sharp (see Babin and Vishik [69], Liu [70], and Ziane [71]). (A simpler proof of (174) can be found in Doering \& Gibbon [2].)

Open Question 9. Is the dimension estimate $d_{\text {box }}(\mathscr{A}) \leq c G$ sharp in bounded domains?

There are still other interesting open questions regarding the dimension of Navier-Stokes attractors, in particular obtaining bounds for physically relevant problems (or at least two-dimensional versions of them). 
6.4. Reflecting the Lengthscales in the Forcing. Note that $G$ reflects only the amount of energy being put into the flow and says nothing of the scales at which the energy is supplied. The following simple calculation, inspired by the paper of Olson \& Titi [72], shows that it is in fact possible to improve on the dimension estimate in the Dirichlet boundary condition case, as well as on the estimate (174) in the periodic case when the forcing is at very small scales, by making a small modification to the previous argument (see $[41,73]$ ). If we return to our previous analysis of (95) but estimate the right-hand side differently we can obtain

$$
\frac{1}{2} \frac{\mathrm{d}}{\mathrm{d} t}|u|^{2}+\nu|D u|^{2} \leq\|f\|_{*}|D u|,
$$

then if we use Young's inequality on the right-hand side and take the time average we will obtain

$$
\left\langle|D u|^{2}\right\rangle \leq \frac{\|f\|_{*}^{2}}{v^{2}}=\lambda_{1} \nu^{2} G_{*}^{2},
$$

where now we have defined an alternative Grashof number $G_{*}$ based on $\|f\|_{-1}$, as

$$
G_{*}:=\frac{\|f\|_{-1}}{v^{2} \lambda_{1}^{1 / 2}}
$$

It follows that

$$
d_{\mathrm{box}}(\mathscr{A}) \leq c G_{*} .
$$

Noting that the constant $c$ is the same as that in (162), that this provides an improvement is guaranteed by the following lemma.

Lemma 30. If $f \in L^{2}$ then $G_{*} \leq G$.

Proof. If $f$ has expansion $f=\sum_{j=1}^{\infty} f_{j} w_{j}$, where $w_{k}$ are the eigenfunctions of $A$, then it follows that

$$
\begin{gathered}
|f|^{2}=\sum_{j=1}^{\infty}\left|f_{j}\right|^{2}, \\
\|f\|_{*}^{2}=\left|A^{-1 / 2} f\right|^{2}=\sum_{j=1}^{\infty} \lambda_{j}^{-1}\left|f_{j}\right|^{2} .
\end{gathered}
$$

Since

$$
\frac{\|f\|_{*}^{2}}{|f|^{2}}=\sum_{j=1}^{\infty} \lambda_{j}^{-1} \frac{\left|f_{j}\right|^{2}}{\sum_{i}\left|f_{j}\right|^{2}}
$$

it follows that $\|f\|_{*} \leq \lambda_{1}^{-1 / 2}|f|$, and so

$$
\frac{\|f\|_{*}^{2}}{v^{4} \lambda_{1}} \leq \frac{|f|^{2}}{v^{4} \lambda_{1}^{2}} \text {. }
$$

In the case of periodic boundary conditions, the eigenvalue corresponding to the eigenfunction with spatial dependence $e^{2 \pi i k \cdot x / L}$ is

$$
\lambda_{k}=\frac{4 \pi^{2}|k|^{2}}{L^{2}}
$$

it follows that $\lambda_{k}^{-1 / 2}$, which has the dimension of a length, is essentially the lengthscale on which the eigenfunction varies. Carrying this idea over to the case of Dirichlet boundary conditions, it is a convenient and suggestive shorthand to describe a forcing of the form

$$
f=\sum_{k_{1} \leq j \leq k_{2}} f_{j} w_{j}
$$

as being confined to lengthscales between $\lambda_{k_{1}}^{-1 / 2}$ and $\lambda_{k_{2}}^{-1 / 2}$; it is clear from (180) that for a forcing of this form

$$
\lambda_{k_{2}}^{-1 / 2}\|f\|_{L^{2}} \leq\|f\|_{*} \leq \lambda_{k_{1}}^{-1 / 2}\|f\|_{L^{2}} .
$$

More suggestively one can say that if the forcing is confined to lengthscales between $l_{\min }$ and $l_{\max }$ then

$$
l_{\min }|f| \leq\|f\|_{*} \leq l_{\max }|f| \text {. }
$$

Using $l_{\text {ref }}=\lambda_{1}^{-1 / 2}$ as a reference length, this gives

$$
\frac{l_{\mathrm{min}}}{l_{\mathrm{ref}}} G \leq G_{*} \leq \frac{l_{\mathrm{max}}}{l_{\mathrm{ref}}} G .
$$

Returning to (180), the ratio $\|f\|_{*}^{2} /|f|^{2}$ appears as an average of squared lengthscales, weighted according to the amount of energy injected at each scale. Accordingly, it is natural to define an effective lengthscale of the forcing by

$$
l_{\mathrm{eff}}=\frac{\|f\|_{*}}{|f|},
$$

in which case the relationship

$$
G_{*}=\frac{l_{\text {eff }}}{l_{\text {ref }}} G
$$

is essentially a tautology. (Ratios of successive Sobolev norms are a natural way to define possible lengthscales; for example, a whole hierarchy of lengths is defined in Doering \& Gibbon [2] using (essentially) the ratios of successive norms of the solution $u$.)

Note that (178) therefore implies that the dimension of the attractor is less than one if the forcing is applied at sufficiently small scales. Since the attractor is a compact connected set, this in fact implies that the attractor is a point [56], so that the dynamics are trivial (no matter how 'complicated' the forcing is at these scales).

6.5. Physical Interpretation of the Attractor Dimension. One way of interpreting the physical significance of an attractor is as a means of giving a rigorous notion of the number of 
independent "degrees of freedom" of the system, a notion introduced by Landau \& Lifshitz [74].

They supposed that there is a smallest physically relevant length-scale $\ell$ in the problem, so that interactions on scales of less than $\ell$ are irrelevant for the dynamics (e.g., in a fluid the viscosity has a large effect on the very small scales, and one might expect that fluctuations on these scales have a negligible effect). A heuristic indication of the number of degrees of freedom would then be given by how many "boxes" of side $\ell$ fit into the volume $\Omega$ within which the system is confined, yielding

$$
n_{\text {heuristic }} \sim|\Omega| \ell^{-d} .
$$

If we assume that this is a good estimate of the number of degrees of freedom, and that this in turn is well estimated by the attractor dimension, we can isolate a length scale, given in terms of $d_{\text {box }}(\mathscr{A})$ by

$$
\ell \sim\left(\frac{|\Omega|}{d_{\mathrm{box}}(\mathscr{A})}\right)^{1 / d}
$$

[cf. [68] or [2]].

Notice that upper bounds on $d_{\text {box }}(\mathscr{A})$ raise the estimate of the length scale $\ell$. Recall that the best current estimate for the dimension of the 2D Navier-Stokes attractor is

$$
d_{\text {box }}(\mathscr{A}) \leq c G^{2 / 3}(1+\log G)^{1 / 3}
$$

in the case of periodic boundary conditions. It is striking that the bound in (191) corresponds via (190) to a length $\ell$ that satisfies

$$
\frac{\ell}{L} \sim G^{-1 / 3}
$$

to within logarithmic corrections ( $L$ denotes the size of one side of our 2D periodic domain). This length scale in (192) is precisely the "Kraichnan length", derived by other (also heuristic) methods as the natural minimum scale in twodimensional turbulent flows [75]. This links the rigorous analytical bound on the attractor dimension with an "intuitive" estimate from fluid dynamics. (The material in this section is discussed in more detail in Robinson [76].)

We will see in Section 8.2.2 that our heuristic derivation of the relationship in (190) can be justified rigorously via an appropriate parametrisation of the attractor, at least when the forcing $f$ is analytic.

\section{Embedding and Parametrisation}

In this section we will state formally a theorem that guarantees that any finite-dimensional set $X$ (and in particular a finite-dimensional global attractor) can be embedded into $\mathbb{R}^{k}$ if $k$ is large enough (roughly twice the dimension of $X$ ). This result can be considered in two ways:

(i) we can take the set $X$ "out" of the infinite-dimensional space and map it, using some linear map $L$, homeomorphically onto a subset of $\mathbb{R}^{k}$; (ii) $L^{-1}$ provides a way of parametrising the attractor using a finite set of coordinates.

We will make use of both interpretations in what follows.

The embedding theorem we give below is due essentially to Hunt \& Kaloshin [77]. The first such embedding result for finite-dimensional sets was proved by Mañé [60]. BenArtzi et al. [78] showed that the inverse of the projection is Hölder continuous in the finite-dimensional case, along with strict bounds on the Hölder exponent. Foias \& Olson [79] subsequently proved that the inverse of the projections is Hölder continuous in the infinite-dimensional case, but without bounds on the Hölder exponent, and Hunt \& Kaloshin introduced the notion of the "thickness" of a set and gave the strict bounds on the exponent given in the following theorem.

The Lipschitz deviation, used in the statement of the theorem given here, was introduced by Olson \& Robinson [80], with the definition revised and investigated further by Pinto de Moura \& Robinson [81].

Definition 31. Let $X$ be a compact subset of a real Hilbert space $H$. Let $\delta_{m}(X, \epsilon)$ be the smallest dimension of a linear subspace $U \subset H$ such that

$$
\operatorname{dist}\left(X, \mathbf{G}_{U}[\Phi]\right)<\epsilon,
$$

for some $m$-Lipschitz function $\Phi: U \rightarrow U^{\perp}$; that is,

$$
\|\Phi(u)-\Phi(v)\| \leq m\|u-v\| \quad \forall u, v \in U,
$$

where $U^{\perp}$ is orthogonal complement of $U$ in $H$ and $\mathbf{G}_{U}[\Phi]$ is the graph of $\Phi$ over $U$,

$$
\mathbf{G}_{U}[\Phi]=\{u+\Phi(u): u \in U\}
$$

Let

$$
\operatorname{dev}_{m}(X)=\limsup _{\epsilon \rightarrow 0} \frac{\log \delta_{m}(X, \epsilon)}{-\log \epsilon} ;
$$

the Lipschitz deviation of $X$ is given by

$$
\operatorname{dev}(X)=\lim _{m \rightarrow \infty} \operatorname{dev}_{m}(X) .
$$

Note that one could make a simpler definition by not allowing the extra flexibility that comes from including the function $\Phi$, that is, measuring only how well $X$ can be approximated by linear subspaces $U$. This quantity was introduced by Hunt \& Kaloshin and termed the "thickness exponent" of $X, \tau(X)$.

Theorem 32 (after Hunt \& Kaloshin). If $X$ is a compact subset of a Hilbert space $H$ then, provided that $k$ is an integer with $k>2 d_{\text {box }}(X)$ and

$$
\theta<\frac{k-2 d_{\text {box }}(X)}{k(1+(\operatorname{dev}(X) / 2))},
$$

a dense set of bounded linear maps from $H$ into $\mathbb{R}^{k}$ have the following properties:

(i) they are injective on $X$, 
(ii) their inverse is Hölder continuous from $L X$ into $X$ with exponent $\theta$,

$$
\left\|L^{-1} x-L^{-1} y\right\| \leq C|x-y|^{\theta} \quad \forall x, y \in L X .
$$

(In fact a "prevalent" set of linear maps are injective with Hölder inverse; see Hunt et al. [82]. One can prove a similar result for subsets of Banach spaces; see Robinson [83].)

It is relatively easy to show that if $X$ is a compact subset of $L^{2}(\Omega)$ that is bounded in $H^{k}(\Omega)$ then $\tau(X) \leq d / k$ when $\Omega \subset \mathbb{R}^{d}$; see Friz \& Robinson [84]. Since $\operatorname{dev}(X) \leq \tau(X)$, this gives a class of sets ("smooth attractors", i.e., those bounded in $H^{k}$ for every $k$ ) that have zero thickness and, hence, zero Lipschitz deviation.

We have shown that when $f$ is smooth the attractor of the 2D Navier-Stokes equations with forcing $f$ consists of smooth functions (Corollary 21), so we immediately have the following result.

Lemma 33. If $f$ is smooth then $\operatorname{dev}(\mathscr{A})=\tau(\mathscr{A})=0$, where $\mathscr{A}$ is the attractor of the $2 \mathrm{D}$ Navier-Stokes equations with forcing $f$.

A natural conjecture, due to Ott et al. [85], is that 'many of the attractors associated with the evolution equations of mathematical physics have thickness exponent zero' Specialising this to the Navier-Stokes equations, one has the following interesting question.

Open Question 10. For less regular $f$, does the attractor of the two-dimensional Navier-Stokes equations have zero thickness?

While this question is open, the Lipschitz deviation can be shown to be zero for the attractors of a very large class of PDEs, as we will see in Section 9.4.

Note that even when a set has zero Lipschitz deviation, Theorem 32 only guarantees that one can find a linear map whose inverse is Hölder continuous with any exponent strictly less than one. This is not ideal, for many reasons. Later, in Section 10, we will consider the problem of constructing a finite-dimensional dynamical system that reproduces the dynamics on the attractor, and this turns out to be critical. But also one might naturally try to compute various quantities that depend on lengths of vectors (e.g., Lyapunov exponents) from observations of $L \mathscr{A}$, and these will be distorted unless the inverse of $L$ is Lipschitz.

The bi-Lipschitz embedding problem, to find conditions that guarantee the existence of an embedding with Lipschitz inverse, has attracted much attention (particularly in the theory of metric spaces; see Heinonen [86]), and is still open.

Open Question 11. What assumptions are required on a set $X \subset H$, or more generally a metric space $(X, d)$, to guarantee the existence of a bi-Lipschitz embedding of $X$ into some $\mathbb{R}^{k}$ ?

(Olson and Robinson showed in [80] that if $X-X$ is a subset of a Hilbert space with finite Assouad dimension then one can find an embedding that is bi-Lipschitz to within logarithmic corrections; that a similar result holds for subsets of Banach spaces was shown by Robinson [83]. See Robinson [58] for further discussion.)

7.1. Time-Delayed Observations. Theorem 32 provides "abstract" embeddings of a finite-dimensional attractor into a finite-dimensional space. But in an experimental situation one would rather be able to take a more specific measurement than some general linear map.

In 1981 Takens showed that for a smooth iterated map $\Phi$ (satisfying some generic assumptions) on smooth finitedimensional manifold $M$, the repeated observations

$$
\left(h(x), h(\Phi(x)), \ldots, h\left(\Phi^{k-1}(x)\right)\right)
$$

provide a one-to-one mapping of $M$ into $\mathbb{R}^{k}$ for a generic set of $h$, provided that $k>2 \operatorname{dim}(M)$.

The following theorem generalises this result to allow fractal subsets of infinite-dimensional spaces. The key element of the proof is a result by Sauer et al. [87] that treats the same situation for fractal subsets of finite-dimensional spaces.

Theorem 34 (see [88]). Let $\mathscr{A}$ be a compact subset of a Hilbert space $H$ with upper box-counting dimension $D$ and thickness exponent zero. Suppose that $\mathscr{A}$ is an invariant set for a Lipschitz map $\Phi: H \rightarrow H$. Fix an integer $k>2 D$ and suppose further that the set $\mathscr{A}_{p}$ of p-periodic points of $\Phi$ satisfies $d_{\text {box }}\left(\mathscr{A}_{p}\right)<$ $p / 2$ for all $p=1, \ldots, k$. Then a prevalent set of Lipschitz maps $f: H \rightarrow \mathbb{R}$ make the $k$-fold observation map $\mathcal{O}: H \rightarrow \mathbb{R}^{k}$

$$
u \longmapsto\left(f(u), f(\Phi(u)), \ldots, f\left(\Phi^{k-1}(u)\right)\right)
$$

one-to-one between $\mathscr{A}$ and its image.

Proof (Sketch). Sauer et al. [87] proved the same result for a compact set $X \subset \mathbb{R}^{N}$ and a Lipschitz map $g: X \rightarrow X$. Their proof can be generalised to the case of a map $g: X \rightarrow X$ such that $g^{r}$ is a $\theta$-Hölder function for any $r \in \mathbb{N}$, provided $k>2 D / \theta$ and the set $X_{p}$ of $p$-periodic points of $g$ satisfies $d_{\text {box }}\left(X_{p}\right)<p / 2 \theta$ for all $p=1, \ldots, k$.

We now combine this with the embedding result of Theorem 32. If $L: H \rightarrow \mathbb{R}^{N}$ provides an embedding $X=L \mathscr{A}$ of $\mathscr{A}$ into $\mathbb{R}^{N}$, note that the induced map $g$ on $X$ is given by $g=L \circ \Phi \circ L^{-1}$. In particular, $g^{r}=L \circ \Phi^{r} \circ \Phi^{-1}$ and $g^{r}$ is $\theta$-Hölder for every $r \in \mathbb{N}$.

Note that while we have obtained an embedding of $\mathscr{A}$ into $\mathbb{R}^{k}$, the result as stated, unlike that of Theorem 32, says nothing about the continuity of the resulting parametrisation of $\mathscr{A}$. Such results are important if one wishes to obtain information about properties of the original dynamical system from measurements via $\mathcal{O}$.

Open Question 12. Can one improve Theorem 34 to obtain information about the continuity of $\mathcal{O}^{-1}$ ? 


\section{Determining Nodes and Parametrisation by Nodal Values}

In this section we return to the particular example of the $2 \mathrm{D}$ Navier-Stokes equations and introduce a different approach to finite-dimensional behaviour, in a form due first to Foias \& Temam [89]. We show that if two solutions converge as $t \rightarrow$ $\infty$ on a sufficiently large collection of points (or "nodes"), then they must converge throughout the domain. This collection of points is referred to as a set of "determining nodes."

It is as important to stress what such results do not say as what they do say. If you take two solutions of the full equations and know that they converge on some set of nodes, you can deduce that the full solutions are converging. However, it does not say a priori that the knowledge of the nodal values at any instant will determine the full solution.

However, it is natural to conjecture (as did Foias \& Temam in [89]) that if one restricts to the attractor-that is, if one has already taken a "dynamical limit" as $t \rightarrow \infty$-then in fact the instantaneous value of the solution at a sufficient number of points will be enough to distinguish different solutions. We prove a generalised version of a result due to Foias \& Titi [90], which in the context of the Navier-Stokes equations is conditional, that guarantees this, and we state a result due to Friz and Robinson [53] that this is indeed the case when the forcing $f$ is analytic.

8.1. Determining Nodes. We choose a finite set of $N$ points, or nodes, in $\Omega=[0, L]^{2}, \mathcal{N}=\left\{x_{j}\right\}_{j=1}^{N}$, and set

$$
d(\mathcal{N})=\sup _{x \in \Omega} \min _{j}\left|x-x_{j}\right|
$$

so that for every $x \in \Omega$ there is an $x_{j}$ such that

$$
\left|x-x_{j}\right| \leq d(\mathcal{N}) \text {. }
$$

We say that $\mathcal{N}$ is a set of determining nodes, if, whenever

$$
\sup _{j}\left|u\left(x_{j}, t\right)-v\left(x_{j}, t\right)\right| \longrightarrow 0,
$$

we have

$$
\sup _{x \in \Omega}|u(x, t)-v(x, t)| \longrightarrow 0 .
$$

Since, by Agmon's inequality in 2D,

$$
\|u\|_{\infty} \leq c|u||A u|
$$

and we remarked at the end of Section 5.2 that there is an absorbing set in $D(A)$, it suffices to show that

$$
|u(t)-v(t)| \longrightarrow 0
$$

In fact, we will show that

$$
|D u(t)-D v(t)| \longrightarrow 0,
$$

which clearly gives (207) (and, hence, (205)) since $|u| \leq$ $\lambda^{-1 / 2}|D u|$.

Fundamental to the proof is the following lemma, relating a bound on $\|w\|_{\infty}$ to a bound on $\left.w\right|_{\mathcal{N}}$.
Lemma 35. If $w \in D(A)$ and one sets

$$
\eta(w)=\max _{x_{j} \in \mathcal{N}}\left|w\left(x_{j}\right)\right|
$$

then

$$
\|w\|_{\infty} \leq \eta(w)+c d(\mathcal{N})^{1 / 2}|A w|
$$

Proof. Recall the Sobolev embedding theorem $H^{2}(\Omega) \quad \subset$ $C^{0,1 / 2}(\bar{\Omega})$, where $C^{0,1 / 2}(\bar{\Omega})$ is the set of continuous functions on $\bar{\Omega}$ with Hölder exponent one half; since $D(A) \subset H^{2}$ and

$$
\|u\|_{H^{2}} \leq c|A u|
$$

(this follows straightforwardly in the case of periodic boundary conditions) we have

$$
|w(x)-w(y)| \leq c|A w||x-y|^{1 / 2}
$$

The expression (210) follows immediately from this and the definition of $d(\mathcal{N})$ and $\eta(w)$.

We will also need the following simple lemma.

Lemma 36. Suppose that $X \in L_{\mathrm{loc}}^{1}(0, \infty)$ is nonnegative and satisfies

$$
\frac{d X}{d t}+a X \leq b(t)
$$

where $b(t) \rightarrow 0$ and that $X(t) \leq k$ for all $t \geq t_{0}$. Then $X(t) \rightarrow$ 0 as $t \rightarrow \infty$.

Proof. Choose $\epsilon>0$. Then there exists a $T$ such that $b(t) \leq$ $\epsilon / 2$ for all $t \geq T$. So for $t \geq T$,

$$
\frac{\mathrm{d} X}{\mathrm{~d} t}+a X \leq \frac{\epsilon}{2}
$$

By Gronwall's inequality,

$$
X(T+t) \leq X(T) e^{-a t}+\frac{\epsilon}{2},
$$

and so choosing $\tau$ large enough that

$$
k e^{-a \tau}<\frac{\epsilon}{2}
$$

we have

$$
X(t) \leq \epsilon \quad \forall t \geq T+\tau,
$$

so that $X(t) \rightarrow 0$.

We now use these two results to study the time evolution of $|D w(t)|$, where $w(t)=u(t)-v(t)$.

Theorem 37 (determining nodes). There exists $\delta>0$ such that if $d(\mathcal{N})<\delta$, then $\mathcal{N}$ are a set of determining nodes. 
Proof. The equation for $w(t)=u(t)-v(t)$ is

$$
\frac{\mathrm{d} w}{\mathrm{~d} t}+v A w+B(u, w)+B(w, u)-B(w, w)=0,
$$

and taking the inner product of this with $A w$ and using $B(u, u, A u)=0(15)$ and the three-term identity

$$
(B(u, w), A w)+(B(w, u), A w)+(B(w, w), A u)=0
$$

which follows by differentiating, we obtain

$$
\frac{1}{2} \frac{\mathrm{d}}{\mathrm{d} t}|D w|^{2}+\nu|A w|^{2}=(B(w, w), A u) .
$$

Thus

$$
\begin{aligned}
\frac{1}{2} \frac{\mathrm{d}}{\mathrm{d} t}|D w|^{2}+\nu|A w|^{2} \leq & \|w\|_{\infty}|D w||A u| \\
\leq & {\left[\eta(w)+c L^{1 / 2} d(\mathcal{N})^{1 / 2}|A w|\right]|D w||A u| } \\
\leq & \eta(w)|D w||A u| \\
& +c L^{1 / 2} d(\mathcal{N})^{1 / 2} \lambda_{1}^{-1 / 2}|A w|^{2}|A u|,
\end{aligned}
$$

so therefore

$$
\begin{aligned}
& \frac{1}{2} \frac{\mathrm{d}}{\mathrm{d} t}|D w|^{2}+\left[\nu-c \lambda_{1}^{-1 / 2} L^{1 / 2} d(\mathcal{N})^{1 / 2}|A u|\right] \lambda_{1}|D w|^{2} \\
& \quad \leq \eta(w)|D w||A u|
\end{aligned}
$$

Now, we know that we have absorbing sets in $V$ and $D(A)$, so that for large enough $t$

$$
|A u| \leq R_{D} \quad\|w\| \leq 2 R_{V},
$$

and therefore

$$
\begin{aligned}
& \frac{1}{2} \frac{\mathrm{d}}{\mathrm{d} t}\|w\|^{2}+\left[\nu-c \lambda^{1 / 2} L^{1 / 2} R_{D} d(\mathcal{N})^{1 / 2}\right] \lambda_{1}\|w\|^{2} \\
& \leq 2 R_{V} R_{D} \eta(w) .
\end{aligned}
$$

Now, choose $\delta$ such that

$$
\mu=v-c \lambda_{1}^{1 / 2} L^{1 / 2} R_{D} \delta^{1 / 2}>0
$$

Then we have

$$
\frac{1}{2} \frac{\mathrm{d}}{\mathrm{d} t}\|w\|^{2}+\mu\|w\|^{2} \leq 2 R_{V} R_{D} \eta(w) .
$$

By assumption, we know that $\eta(w) \rightarrow 0$, and we also know that for $t$ large enough $\|w(t)\|^{2} \leq 4 R_{V}^{2}$.

It follows from Lemma 36 that $\|w(t)\|^{2} \rightarrow 0$ as $t \rightarrow \infty$, and so $\|w(t)\|_{\infty} \rightarrow 0$ and the nodes $\mathcal{N}$ are determining.

Here we have made no attempt to obtain the best estimate for the separation $\delta$, and indeed, the estimate derived from (225) is very coarse. The bound was improved in a series of papers, Foias \& Titi [90] and Jones \& Titi [91], until the best current results which are due to Jones \& Titi [92] (their paper gives the best bounds for determining nodes, modes, and volume elements (see next section)), as

$$
\delta \leq c G^{-1 / 2}
$$

compare this with the length scale from the Kraichnan theory discussed in Section 6.5, where

$$
\left(\frac{L_{\chi}}{L}\right) \sim G^{-1 / 3} \text {. }
$$

This motivates the following.

Open Question 13. Can one obtain a bound on the number of determining nodes that agrees with the attractor dimension estimate, that is, $\delta \sim G^{-1 / 3}$ ?

8.2. Nodal Parametrisation. As remarked earlier, Foias \& Temam [89] conjectured that on the attractor one should be able to take a finite number of nodes and distinguish functions by their value at these nodes at one fixed time; that is, for a set $\left\{x_{j}\right\}_{j=1}^{k} \in \Omega$, if $u, v \in \mathscr{A}$ then

$$
\begin{gathered}
u\left(x_{j}\right)=v\left(x_{j}\right) \quad \forall j=1, \ldots, k \\
\Longrightarrow u=v .
\end{gathered}
$$

8.2.1. A Conditional Result. The first result in this direction was due to Foias \& Titi [90], who showed, using the existence of an inertial manifold (see Section 9.2), that the result holds for the one-dimensional Kuramoto-Sivashinsky equation.

We give a version of their argument here which is valid for the $2 \mathrm{D}$ Navier-Stokes equations, but which requires a strong assumption which is not known to be valid in this case. The main assumption of the lemma is that for some $\Lambda>0$,

$$
|A u-A v| \leq \Lambda|u-v| \quad \forall u, v \in \mathscr{A} .
$$

Combining this with (210) it follows that if $x$ is contained in a cube centred at $y$ with sides of length $\delta$ then

$$
|w(x)-w(y)|^{2} \leq \frac{c^{2}}{2}\|A w\|_{L^{2}}^{2} \delta \leq \frac{c^{2}}{2} \Lambda^{2}\|w\|_{L^{2}}^{2} \delta .
$$

We will return to assumption (230) in Section 9.3. (For the 1D Kuramoto-Sivashinsky equation, the existence of an inertial manifold guarantees that $A^{1 / 2}$ is Lipschitz on the attractor and on a one-dimensional domain functions in $H^{1}$ are 1/2-Hölder, so the conclusion holds without the need for additional assumptions in this case. A more general result is given by Cockburn et al. [93].)

Lemma 38. Consider the 2D Navier-Stokes equations, and suppose that there exists a constant $\Lambda>0$ such that

$$
\|A u-A v\|_{L^{2}} \leq \Lambda\|u-v\|_{L^{2}} \quad \forall u, v \in \mathscr{A} .
$$

Suppose that $\delta$ is sufficiently small that

$$
|\Omega|^{1 / 2} c \Lambda \delta<1,
$$


where $c$ is the constant in (210), and let $\mathbf{x}=\left\{x_{j}\right\}_{j=1}^{N}$ be a collection of nodes placed at the corners of squares with sides of length $\delta$. Then the map $u \mapsto E_{\mathbf{x}}$, where

$$
E_{\mathbf{x}}(u)=\left(u\left(x_{1}\right), \ldots, u\left(x_{N}\right)\right),
$$

is a bi-Lipschitz embedding of $\mathscr{A}$ into $\mathbb{R}^{N}$, and in particular the nodes $\left\{x_{j}\right\}$ are instantaneously determining.

Proof. Suppose that $u, v \in \mathscr{A}$ and set $w=u-v$. Let $\mathbf{x}$ be a collection of $N$ nodes equally spaced by $\delta$; then certainly $N \leq 2|\Omega| \delta^{-n}$. Noting that

$$
\begin{aligned}
\left|E_{\mathbf{x}}(u)-E_{\mathbf{x}}(v)\right| & =\left|E_{\mathbf{x}}(w)\right| \\
& \leq N^{1 / 2}\|w\|_{\infty} \leq c \Lambda N^{1 / 2}|w|,
\end{aligned}
$$

it follows that $E_{\mathbf{x}}: \mathscr{A} \rightarrow \mathbb{R}^{k}$ is Lipschitz.

Now we show the reverse inequality for $\delta$ sufficiently small. Split $\Omega$ into a collection of $N=|\Omega| \delta^{-2}$ squares $\left\{Q_{j}\right\}$ with sides of length $\delta$. As in (231) for any $x \in Q_{j}$ we have

$$
\left|w(x)-w\left(x_{j}\right)\right| \leq \frac{c}{\sqrt{2}}\|w\|_{H^{2}} \delta,
$$

and so

$$
\begin{aligned}
|w(x)|^{2} & \leq 2\left|w\left(x_{j}\right)\right|^{2}+c^{2}\|w\|_{H^{2}}^{2} \delta^{2} \\
& \leq 2\left|w\left(x_{j}\right)\right|^{2}+c^{2} \Lambda^{2}\|w\|_{L^{2}}^{2} \delta^{2}
\end{aligned}
$$

for any $x \in Q_{j}$. Therefore

$$
\begin{aligned}
\|w\|_{L^{2}}^{2} & =\int_{\Omega}|w(x)|^{2} \mathrm{~d} x \\
& \leq \sum_{j} \int_{Q_{j}}|w(x)|^{2} \mathrm{~d} x \\
& \leq \sum_{j} \int_{Q_{j}}\left(2\left|w\left(x_{j}\right)\right|^{2}+c^{2} \Lambda^{2}\|w\|_{L^{2}}^{2} \delta^{2}\right) \\
& =2 \delta^{2} \sum_{j}\left|w\left(x_{j}\right)\right|^{2}+c^{2} \Lambda^{2}\|w\|_{L^{2}}^{2} N \delta^{4} \\
& =2 \delta^{2}\left|E_{\mathbf{x}}(w)\right|^{2}+|\Omega| \delta^{2} c^{2} \Lambda^{2}\|w\|_{L^{2}}^{2} ;
\end{aligned}
$$

thus, if $\delta$ is sufficiently small that (233) is satisfied then

$$
\|u-v\|_{L^{2}} \leq C\left|E_{\mathbf{x}}(u-v)\right|
$$

for some $C>0$, and, hence, $E_{\mathbf{x}}(u)=E_{\mathbf{x}}(v)$ implies that $u=$ $v$.

8.2.2. Nodal Parametrisation for Analytic $f$. One can also parametrise the attractor by nodal values provided that the attractor consists of analytic functions, with $k \sim d_{\text {box }}(\mathscr{A})$, for almost every choice of $k$ points in $\Omega$. This result, originally due to Friz \& Robinson [53], has been considerably refined and the most powerful version is given in Kukavica \& Robinson [94]. We only state the result here, but note that its proof makes use of the attractor, its finite-dimensionality, and the regularity of its elements.
Theorem 39. Let $\mathscr{A}$ be a compact subset of $L^{2}\left(\Omega, \mathbb{R}^{d}\right)$ with finite dimension $d_{\mathrm{box}}(\mathscr{A})$ that consists of real analytic functions. Then for $k \geq 16 d_{\text {box }}(\mathscr{A})+1$ almost every set $\mathbf{x}=$ $\left(x_{1}, \ldots, x_{k}\right)$ of $k$ points in $\Omega$ makes the map $E_{\mathbf{x}}$, defined by

$$
E_{\mathbf{x}}[u]=\left(u\left(x_{1}\right), \ldots, u\left(x_{k}\right)\right)
$$

one-to-one between $X$ and its image.

We note here that for the 2D Navier-Stokes equations, if we space our nodes evenly over the domain, then the separation required by our theorem is of the order of $G^{-1 / 3}$, with logarithmic corrections, confirming the Kraichnan length scale by analytically rigorous means. Note also that this is an entirely natural way to produce a length-scale from the equations and ties in with the heuristic definition of the "number of degrees of freedom" due to Landau \& Lifshitz, which indicates that one would expect that

$$
d_{\text {box }}(\mathscr{A}) \sim\left(\frac{L}{L_{\chi}}\right)^{2},
$$

cf. Section 6.5 .

Note that since one can show (under minimal assumptions) that these "instantaneous" determining nodes are "determining" in the sense of Theorem 37 (see [53]), this shows that when $f$ is analytic the answer to Open Question 13 is positive.

It is natural to ask whether the result of Theorem 39 can be significantly improved.

Open Question 14. Can one parametrise finite-dimensional sets of functions by nodal values under weaker conditions?

As a first step away from analyticity, note that Kukavica \& Robinson [94] showed using a more general version of Theorem 39 based on functions with finite order of vanishing and arguments essentially due to Poon $[95,96]$ that such a parametrisation occurs for attractors of reaction-diffusion equations when the nonlinearity is only $C^{\infty}$. It is natural to ask whether a similar result holds for the Navier-Stokes equations.

Open Question 15. Can one parametrise the attractor of the 2D NSE by nodal values when $f$ is only $C^{\infty}$ (and not necessarily analytic)?

\section{Determining Modes and the Foias-Temam Conjecture}

In a paper from 1967, Foias \& Prodi [44] showed that the dynamics are "determined" by a finite number of Fourier modes, in that if $u(t)$ and $v(t)$ are two solutions of the NavierStokes equations, and for $N$ sufficiently large

$$
\left|P_{N} u(t)-P_{N} v(t)\right| \longrightarrow \infty
$$

then in fact $|u(t)-v(t)| \rightarrow \infty$. 
Just as with the "determining nodes" discussed in the previous section, it is important that this result does not say that the solutions of the $P$-mode Galerkin truncation determine the solution, nor that knowledge of the $P$ modes at any instant will determine the full solution.

While the "natural conjecture" in this case is that on the attractor a sufficiently high-dimensional finite-dimensional Fourier projection will distinguish different elements of the attractor $\left(P_{N} u=P_{N} v \Longrightarrow u=v\right)$ this is still open.

9.1. Determining Modes. We give a simple proof that enough modes are determining, following Foias et al. [97].

Theorem 40 (Determining modes). There exists a $N_{0}$ such that the first $N$ Fourier modes are determining provided that $N>N_{0}$.

Proof. If $u$ and $v$ are two solutions then the equation satisfied by $w=u-v$ is

$$
\frac{\mathrm{d} w}{\mathrm{~d} t}+v A w+B(w, u)+B(v, w)=0 .
$$

We write $p=P_{n} w$ and $q=Q_{n} w$; take the inner product of this equation with $q$ to obtain

$$
\frac{1}{2} \frac{\mathrm{d}}{\mathrm{d} t}|q|^{2}+v|D q|^{2}+(B(w, u), q)+(B(v, p), q)=0,
$$

since $w=p+q$ and $(B(u, q), q)=0$. Therefore

$$
\begin{aligned}
& \frac{1}{2} \frac{\mathrm{d}}{\mathrm{d} t}|q|^{2}+v|D q|^{2} \\
& \leq|(B(q, u), q)|+|(B(p, u), q)|+|(B(v, p), q)| \\
& \leq c\|q\|_{L^{4}}^{2}|D u|+\|p\|_{L^{\infty}}|D u||q|+\|v\|_{L^{4}}|D q|_{L^{4}}|p| \\
& \leq c|q||D q||D u|+c|p|^{1 / 2}|A p|^{1 / 2}|D u||q| \\
& \quad+c|v|^{1 / 2}|D v|^{1 / 2}|D p|^{1 / 2}|A p|^{1 / 2}|q| \\
& \leq c R_{1} \lambda_{n+1}^{-1 / 2}|D p|^{2}+c R_{0} R_{1} \lambda_{n}^{1 / 2}|p| \\
& \quad+c R_{0}^{3 / 2} R_{1}^{1 / 2} \lambda_{n}^{3 / 4}|p|,
\end{aligned}
$$

and so, since $|D q|^{2} \geq \lambda_{n+1}|q|^{2}$, this becomes

$$
\begin{aligned}
& \frac{1}{2} \frac{\mathrm{d}}{\mathrm{d} t}|q|^{2}+\lambda_{n+1}\left[\nu-c R_{1} \lambda_{n+1}^{-1 / 2}\right]|q|^{2} \\
& \quad \leq\left[c R_{0} \mathrm{R}_{1} \lambda_{n}^{1 / 2}+c R_{0}^{3 / 2} R_{1}^{1 / 2} \lambda_{n}^{3 / 4}\right]|p| .
\end{aligned}
$$

If $n$ is sufficiently large that $c R_{1} \lambda_{n+1}^{-1 / 2}<\nu$ then, since we have assumed that $|p(t)| \rightarrow 0$ as $t \rightarrow \infty$, this is an equation of the form $\dot{X}+a X \leq b(t)$ as in the proof of Theorem 37; using Lemma 36 it follows that $|q(t)| \rightarrow 0$ as $t \rightarrow \infty$, and the proof is complete.

Note that one can obtain a similar result by taking the average the solution over a subgrid of a set of smaller squares, termed "determining volume elements" (introduced in [90]; see also $[92,98])$. So, we split $\Omega$ into $N$ equal squares, of sides $L / \sqrt{N}$, and label them $Q_{j}, j=1, \ldots, N$. We define the average of $u(x, t)$ over a square as

$$
\langle u\rangle_{Q_{j}}=\frac{N}{L^{2}} \int_{Q_{j}} u(x) d x
$$

and show that if $N$ is "large enough", then

$$
\sup _{j}\left|\langle u(x, t)-v(x, t)\rangle_{Q_{j}}\right| \longrightarrow 0
$$

implies that

$$
\sup _{x \in \Omega}|u(x, t)-v(x, t)| \longrightarrow 0 .
$$

A unified treatment of determining nodes, modes, volume elements, and more general "determining functionals" is given in Cockburn et al. [93].

9.2. Inertial Manifolds. One situation in which a Fourier projection will distinguish elements of the attractor is when the equation admits an inertial manifold. An inertial manifold $\mathscr{M}$ for a general evolution equation

$$
\frac{\mathrm{d} u}{\mathrm{~d} t}+A u=f(u)
$$

is a finite-dimensional, positively invariant Lipschitz smooth manifold that exponentially attracts all trajectories $[99,100]$, so that

$$
S(t) \mathscr{M} \subset \mathscr{M} \quad \operatorname{dist}\left(S(t) u_{0}, \mathscr{M}\right) \leq C\left(u_{0}\right) \mathrm{e}^{-k t} .
$$

In order to describe the results further, we assume that $A$ is a positive, linear, self-adjoint operator with compact inverse, and $f$ is a Lipschitz function from $D\left(A^{\alpha}\right)$ (the domain of $A^{\alpha}$ ) into $H$, for some $0 \leq \alpha<1$. Since $A$ has a compact inverse, there is a set of orthonormal eigenfunctions $\left\{w_{n}\right\}$ of $A$ with corresponding eigenvectors $\lambda_{n}$, which one can order such that

$$
A w_{n}=\lambda_{n} w_{n} \quad \lambda_{n+1} \geq \lambda_{n} \quad \lambda_{n} \longrightarrow \infty ;
$$

see Renardy \& Rogers [101], for example. One can define the finite-dimensional projection operators $P_{n}$ and their orthogonal complements $Q_{n}$ by

$$
P_{n} u=\sum_{1}^{n}\left(u, w_{j}\right) w_{j} \quad Q_{n} u=\sum_{n+1}^{\infty}\left(u, w_{j}\right) w_{j},
$$

where $(\cdot, \cdot)$ is the scalar product in $H$.

All current existence proofs give the inertial manifold as a Lipschitz (or smoother) graph over one of the finitedimensional subspaces $P_{n} H$; that is,

$$
\mathscr{M}=\left\{p+\phi(p): p \in P_{n} H\right\} .
$$

For a general evolution equation

$$
\frac{\mathrm{d} u}{\mathrm{~d} t}+A u=f(u),
$$


restricting the flow from (255) to the manifold given by (254) immediately yields the set of ordinary differential equations for $p=P u$, as

$$
\frac{\mathrm{d} p}{\mathrm{~d} t}+A p=P f(p+\phi(p)) .
$$

Since $p \in P_{n} H \simeq \mathbb{R}^{n}$ and $\phi$ is Lipschitz, it follows that (256) has unique solutions (see Hartman [102], Section II, Theorem 1.1). Clearly the solutions of (256) on $P_{n} \mathscr{A}$ are precisely those projected down from $\mathscr{A}$; that is,

$$
p(t)=P_{n} S(t)[p(0)+\phi(p(0))],
$$

and since $\mathscr{M}$ is an invariant manifold in $H, P_{n} \mathscr{A}$ is the global attractor for the finite-dimensional system (256).

However, there are two problems with the inertial manifold approach. Outstandingly, the conditions known to be sufficient to prove the existence of such an object are restrictive; essentially a large gap is required in the spectrum of the linear operator $A$,

$$
\lambda_{n+1}-\lambda_{n}>C \lambda_{n+1}^{\alpha}
$$

Although this is satisfied for some interesting examples (e.g., the Kuramoto-Sivashinsky equation [18, 103, 104], the Ginzburg-Landau equation [18], and reaction-diffusion equations in space dimension 1 [18] (and some special domains in dimensions 2 and 3, Mallet-Paret \& Sell [105]), there are many situations in which one can prove the existence of a finite-dimensional global attractor but not (at present) of an inertial manifold-of greatest interest, perhaps, are the 2D Navier-Stokes equations.

The problem here is that the eigenvalues of the Stokes operator satisfy $\lambda_{n} \sim c n$ (see (159)), and so the gap between consecutive eigenvalues does not grow as $n \rightarrow \infty$.

Open Question 16. Do the 2D Navier-Stokes equations possess an inertial manifold?

However, on the sphere the eigenvalues of the negative Laplacian are $\lambda_{n}=n(n+1)$, and so in this case $\lambda_{n+1}-\lambda_{n}=$ $2(n+1)$. In the case of the $2 \mathrm{D}$ Navier-Stokes equations one can take $\alpha=1 / 2$ in (258), and so the right-hand side also behaves like $n$. In this marginal case one may hope to obtain a positive result.

Open Question 17. Do the 2D Navier-Stokes equations on the sphere possess an inertial manifold?

A second problem is that, the dimension of the inertial manifold and, hence, of the differential system (257) can be much greater than that of the attractor. For example, for the Kuramoto-Sivashinsky equation

$$
u_{t}+u_{x x x x}+u_{x x}+u u_{x}=0 \quad u(x+L, t)=u(x, t)
$$

the best estimate of the dimension of the attractor is $d_{\text {box }}(X) \sim L^{1.275}[18,106]$, whereas the best estimate of the dimension of the inertial manifold is $\operatorname{dim} \mathscr{M} \sim L^{1.64}(\ln L)^{0.2}$ [104].
More generally, one can ask if the whole approach is too restrictive.

Open Question 18. Can one obtain inertial manifolds as bona fide manifolds, that is, not given as graphs?

9.3. The Foias-Temam Conjecture. As we have already discussed, the existence of an inertial manifold for the 2D Navier-Stokes evolution equation is an important open problem and related to the following conjecture due to Foias \& Temam, which provides a weaker version of Open Question 16.

Open Question 19. Does there exist an $n>0$ such that the solutions on the attractor of the 2D Navier-Stokes equations are determined by their first $n$ Fourier modes, that is, if $u, v \in$ $A$ then

$$
P_{n} u=P_{n} v \Longrightarrow u=v ?
$$

We note here the following conditional result that would supply an affirmative answer to this question. We showed in Lemma 38 that the same condition (261) would also guarantee the existence of a set of instantaneous determining nodes.

Proposition 41. Suppose that A is Lipschitz continuous on the attractor

$$
|A u-A v| \leq L|u-v| \quad \forall u, v \in \mathscr{A}
$$

for some $L>0$. Then the attractor is a subset of a Lipschitz manifold given as a graph over $P_{n} H$ for some $n$.

Proof. Write $w=u-v$ for $u, v \in \mathscr{A}$. If $A$ is Lipschitz continuous from $\mathscr{A}$ into $H$ then

$$
|A w| \leq L|w|
$$

for some $L$. Now split $w=P_{n} w+Q_{n} w$, and observe that we have both

$$
\begin{aligned}
|A w|^{2} & =\left|A\left(P_{n} w+Q_{n} w\right)\right|^{2} \\
& =\left|A\left(P_{n} w\right)\right|^{2}+\left|A\left(Q_{n} w\right)\right|^{2} \geq \lambda_{n+1}^{2}\left|Q_{n} w\right|^{2}, \\
|A w|^{2} & \leq L^{2}|w|^{2} \leq L^{2}\left|P_{n} w\right|^{2}+L^{2}\left|Q_{n} w\right|^{2} .
\end{aligned}
$$

Since $\lambda_{n} \rightarrow \infty$ as $n \rightarrow \infty$, we can choose $n$ large enough that $\lambda_{n+1}>L$, and then write

$$
\left(\lambda_{n+1}^{2}-L^{2}\right)\left|Q_{n} w\right|^{2} \leq L^{2}\left|P_{n} w\right|^{2}
$$

that is,

$$
\left|Q_{n} w\right| \leq\left(\frac{L^{2}}{\lambda_{n+1}^{2}-L^{2}}\right)^{1 / 2}\left|P_{n} w\right|
$$

It follows that we can define $\Phi\left(P_{n} u\right)=Q_{n} u$ uniquely for each $u \in \mathscr{A}$, and then

$$
\left|\Phi\left(p_{1}\right)-\Phi\left(p_{2}\right)\right| \leq\left(\frac{L^{2}}{\lambda_{n+1}^{2}-L^{2}}\right)^{1 / 2}\left|p_{1}-p_{2}\right|,
$$

so that the attractor is a subset of a Lipschitz graph over $P_{n} H$. 
One can obtain some continuity of $A$ on $\mathscr{A}$ (but sadly not Lipschitz continuity) by assuming that $\mathscr{A}$ consists of regular functions, as the following simple result shows; if $\mathscr{A}$ is bounded in $D\left(A^{1+r}\right)$ then $A$ is Hölder continuous on $\mathscr{A}$.

Lemma 42. If $\mathscr{A}$ is bounded in $D\left(A^{1+r}\right)$,

$$
\sup _{u \in \mathscr{A}}\left|A^{1+r} u\right| \leq K
$$

then

$$
|A(u-v)| \leq(2 K)^{1 /(1+r)}|u-v|^{r /(1+r)} .
$$

Proof. Setting $w=u-v$ write $w=\sum c_{n} w_{n}$, where $\left\{w_{n}\right\}$ are the eigenfunctions of $A$, and then

$$
|A w|^{2}=\sum_{n=1}^{\infty} \lambda_{n}^{2}\left|c_{n}\right|^{2}
$$

Now use the Hölder inequality with $p=(1+r) / r$ and $q=1+r$, so that

$$
\begin{aligned}
|A w|^{2} & \leq\left(\sum_{n=1}^{\infty}\left|c_{n}\right|^{2}\right)^{r /(1+r)}\left(\sum_{n=1}^{\infty}\left|\lambda_{n}\right|^{2(1+r)}\left|c_{n}\right|^{2}\right)^{1 /(1+r)} \\
& =|w|^{2 r /(1+r)}\left|A^{1+r} w\right|^{2 /(1+r)}
\end{aligned}
$$

which gives (268).

If the attractor is bounded in some Gevrey class, as in Corollary 23, then one can use the resulting bounds $\left|A^{k} u\right|^{2} \leq$ $(4 k) ! /(2 \tau)^{4 k}$ in (268) and minimise with respect to $k$ to deduce that

$$
|\mathrm{A} u-A v| \leq c|u-v|(\log |u-v|)^{2}
$$

for some $c>0$. In the light of Proposition 41 this is somewhat frustrating.

9.4. Zero Lipschitz Deviation. In fact, a stronger result than (271) holds much under weaker conditions. Kukavica [19] showed, using refined methods related to the backwards uniqueness proof of Lemma 5, that on the attractor of the 2D Navier-Stokes equations with periodic boundary conditions and a forcing $f \in H$, that

$$
\left|A^{1 / 2}(u-v)\right|^{2} \leq C|u-v|^{2} \log \left(\frac{M_{1}^{2}}{|u-v|^{2}}\right), \quad \forall u, v \in \mathscr{A},
$$

where $M_{1} \geq 4 \sup _{u \in \mathscr{A}}|u|$.

One can deduce from this [81, 107] that the Lipschitz deviation of the Navier-Stokes attractor is zero, even when we only have $f \in H$.

Proposition 43. If $f \in H$ then $\operatorname{dev}(\mathscr{A})=0$, where $\mathscr{A}$ is the attractor of the $2 \mathrm{D}$ Navier-Stokes equations.
Proof. Let $P_{n}$ be the orthogonal projection onto the first $n$ eigenfunctions of $A$. Consider a subset $X$ of $\mathscr{A}$ that is maximal for the relation

$$
\left|Q_{n}(u-v)\right| \leq\left|P_{n}(u-v)\right| \quad \forall u, v \in X .
$$

For every $p \in P_{n} X$ with $p=P_{n} u, u \in X$, define $\phi_{n}(p)=Q_{n} u$. From (273) this is well defined and

$$
\left|\phi_{n}(p)-\phi_{n}\left(p^{\prime}\right)\right| \leq\left|p-p^{\prime}\right| \quad \forall p, p^{\prime} \in P X .
$$

We can extend $\phi_{n}$ from the closed set $P_{n} X \subset P_{n} H$ to a function $\Phi: P_{n} H \rightarrow Q_{n} H$, preserving the Lipschitz constant [108].

We now show that

$$
\operatorname{dist}(\mathscr{A}, \mathbf{G}[\Phi]) \leq \epsilon_{n}=2 M_{1}^{2} e^{-\lambda_{n+1} / \sqrt{2} C} .
$$

Indeed, if $u \in \mathscr{A}$ but $u \notin X$ then there is a $v \in X$ such that

$$
\left|Q_{n}(u-v)\right| \geq\left|P_{n}(u-v)\right|,
$$

and then, noting that $|w| \leq 2|Q w|$, we have

$$
\begin{aligned}
\lambda n+1\left|Q_{n} w\right|^{2} & \leq|D w|^{2} \\
& \leq 2 C|Q w|^{2} \log \left(\frac{M_{0}^{2}}{|Q w|^{2}}\right),
\end{aligned}
$$

from which $\left|Q_{n} w\right|^{2} \leq \epsilon_{n} / 2$ and (275) follows.

The bound in (275) implies that $\delta_{1}\left(\mathscr{A}, \epsilon_{n}\right)=n$ and hence

$$
\limsup _{n \rightarrow \infty} \frac{\log \delta_{1}\left(\mathscr{A}, \epsilon_{n}\right)}{-\log \epsilon_{n}}=\limsup _{n \rightarrow \infty} \frac{\log n}{\sigma \lambda_{n+1}-\log c_{0}}=0,
$$

since the eigenvalues $\lambda_{n}$ satisfy

$$
\limsup _{n \rightarrow \infty} \frac{\log n}{\lambda_{n}}=0 \text {. }
$$

Thus $\operatorname{dev}(\mathscr{A}) \leq \operatorname{dev}_{1}(\mathscr{A})=0$.

\section{Finite-Dimensional Dynamics?}

Since the attractor can be parametrised by a finite number of parameters and (equivalently) can be "faithfully represented" in a finite-dimensional space $\mathbb{R}^{2 D+1}$, it is a natural question whether one can construct a finite-dimensional dynamical system which has an attractor on which the dynamics are "the same" as those on $\mathscr{A}$. In this sense, the question is whether the dynamics are in some sense "asymptotically finite-dimensional." We can make this precise in the following rather wordy definition.

Definition 44. The dynamics of $S(t)$ are asymptotically finitedimensional if for some $k$, comparable to $d_{\text {box }}(\mathscr{A})$, there exists a map $\varphi: H \rightarrow \mathbb{R}^{k}$ that is injective on $\mathscr{A}$, and a dynamical system $\{T(t)\}_{t \geq 0}$ defined on the whole of $\mathbb{R}^{k}$ such that the dynamics on $\mathscr{A}$ are conjugate to those of the finitedimensional system under $\varphi$; that is,

$$
\left.S(t)\right|_{\mathscr{A}}=\varphi^{-1} \circ T(t) \circ \varphi .
$$


Such issues were first discussed explicitly in Eden et al. [55]. Some partial results can be found in Robinson [16, 109]. Romanov [110] makes a similar definition, dropping the requirement that $X$ is the attractor for $T(t)$, but requiring $\varphi$ to be bi-Lipschitz. He obtains some nice general results about such systems.

Note that this definition does not require

(i) that $T(t)$ is generated by an ordinary differential equation on $\mathbb{R}^{k}$,

(ii) that $\varphi(\mathscr{A})$ is the attractor of the finite-dimensional system,

although both would be ideal.

This question is still entirely open.

Open Question 20. If $S(t)$ has a finite-dimensional attractor, are its dynamics asymptotically finite-dimensional?

There seem to be some major obstructions to proving such a result in continuous time [111], certainly to the "obvious" approach (and refinements), which attempt to obtain $T(t)$ from an appropriate ordinary differential equation. In this approach, take one of the linear maps $L$ from Theorem 32 that embeds $\mathscr{A}$ into $\mathbb{R}^{k}$, and try to use this to write down a finite-dimensional set of ODEs that reproduces the dynamics. If we write the original governing equation as

$$
\frac{\mathrm{d} u}{\mathrm{~d} t}=F(u):=-A u+B(u, u)
$$

then the resulting ODE for $x=L u$ would be

$$
\dot{x}=\operatorname{Lu}=L F(u)=\operatorname{LF}\left(L^{-1} x\right) .
$$

For solutions of this ODE to be unique, standard conditions require $f(x):=L F\left(L^{-1} x\right)$ to be a function such that

$$
|f(x)-f(y)| \leq \omega(|x-y|),
$$

where $\omega$ satisfies $\int_{0}^{1}(1 / \omega(r)) \mathrm{d} r=\infty$ (see Corollary 6.2 in Chapter III of [102]). Taking $\omega(r)=L r$ yields the standard result that a Lipschitz ODE has unique solutions, but this can be weakened by taking $\omega(r)=\operatorname{Lr} \log (1+r)^{\alpha}$ provided that $\alpha \leq 1$.

The continuity of $f$ depends on two factors (since $L$ is bounded and linear, this is not an issue):

(i) the continuity of $F$ on $\mathscr{A}$;

(ii) the continuity of $L^{-1}$.

Usually (and in the 2D Navier-Stokes equations) the continuity of $F$ will be determined by the continuity of $A$ on the attractor, and we have already seen in Lemma 38 and Proposition 41 that Lipschitz continuity of $A$ on the attractor has many consequences. Here, then, is another.

Proposition 45. If $A$ is Lipschitz on the attractor,

$$
|A u-A v| \leq L|u-v| \quad \forall u, v \in \mathscr{A}
$$

then the dynamics are asymptotically finite-dimensional.
Proof. If $A$ is Lipschitz continuous on the attractor then so is $F$, since, writing $w=u-v$,

$$
\begin{aligned}
|B(u, u)-B(v, v)| & \leq|B(u, w)|+|B(w, v)| \\
& \leq\|u\|_{L^{4}}\|\nabla w\|_{L^{4}}+\|w\|_{L^{4}}\|\nabla v\|_{L^{4}} \\
& \leq C|A w| .
\end{aligned}
$$

Proposition 41 shows that the attractor is contained in the graph of a Lipschitz function $\Phi$ over $P_{N} H$ for some $N$. Thus the equation satisfied by $p=P_{N} u$ is

$$
\dot{p}=P_{N} F(p+\Phi(p)),
$$

the right-hand side of which is Lipschitz continuous.

However, such continuity of $A$ is not known. We remarked after the proof of Lemma 42 that one can obtain $\log$-Lipschitz continuity of $A$ on the attractor under the assumption that $f$ is analytic, but with logarithmic exponent 2. Continuity of $A^{1 / 2}$ on the attractor was shown by Kukavica [19] with logarithmic exponent $1 / 2$ (see (272)), and it was shown by Pinto de Moura \& Robinson [107] that a similar argument can be used to show that $A$ is log-Lipschitz with logarithmic exponent 1 . If we could obtain an embedding with Lipschitz inverse then this would be sufficient; but the embedding Theorem 32 only guarantees that $L^{-1}$ is Hölder continuous. Thus even the log-Lipschitz embedding theorem of Olson \& Robinson [80] (which requires strong conditions on $\mathscr{A}$ that are probably not satisfied by the Navier-Stokes attractor) is not sufficient, since it would yield a function $f$ that is log-Lipschitz with logarithmic exponent larger than 1 (see [112] for further discussion of this approach).

Something can be done for iterated homeomorphisms, however. The following result, which uses topological properties of the global attractor, is proved in Robinson \& SánchezGabites [39].

Theorem 46. Let $\mathscr{A}$ be the attractor of a homeomorphism $F: H \rightarrow H$ with $d_{\text {box }}(\mathscr{A}) \leq k$. For any $\epsilon>0$ there exist homeomorphisms

$$
\varphi: \mathscr{A} \longrightarrow A \subset \mathbb{R}^{4 k+4}, \quad f: \mathbb{R}^{4 k+4} \longrightarrow \mathbb{R}^{4 k+4}
$$

such that the dynamics on $\mathscr{A}$ and $A$ are conjugate under $\varphi$, that is,

$$
\left.F\right|_{\mathscr{A}}=\varphi^{-1} \circ f \circ \varphi
$$

and $\left\{f^{n}\right\}$ has an attractor $A_{f}$ with

$$
A \subset A_{f} \subset N(A, \epsilon),
$$

where

$$
N(A, \epsilon)=\left\{y \in \mathbb{R}^{4 k+4}: \operatorname{dist}(y, A)<\epsilon\right\} .
$$

However, even this is not ideal, since it is natural to hope that one could obtain a similar result with $A$ the attractor of $f$.

Open Question 21. Can one prove Theorem 46 but ensure that $A$ is the attractor of $f$ (i.e., $A=A_{f}$ )? 


\section{The 3D Navier-Stokes Equations}

We have concentrated throughout this review on the 2D Navier-Stokes equations, since at present we cannot show that the 3D Navier-Stokes equations generate unique weak solutions, nor that the strong solutions, which are unique, exist for all time. Trying to investigate the existence of attractors without the guarantee of a sensible semigroup seems futile.

However, we end with a result that shows that if we are prepared to assume that the equations generate a semigroup on $V$, that is, if we assume the existence of strong solutions, then we can show that the equations must have a global attractor. In fact the result here just shows the existence of an absorbing set bounded in $V$, and to show that there is a global attractor we would need an absorbing set that is compact in $V$. A relatively straightforward argument can be used to prove the existence of an absorbing set that is bounded in $D(A)$ once we have the absorbing set in $V$, and, hence, of a global attractor.

What we are doing here is making a physically reasonable assumption in a mathematically precise way and then deducing an entirely mathematical consequence. It allows us to consider the asymptotic regimes of the "true" NavierStokes equations and so fully developed turbulence, within a mathematical framework.

Another way to view this theorem, which does not require us to make any "unjustified" assumptions, is as a description of the way in which the 3D Navier-Stokes equations must break down if they are not well posed. The theorem shows that existence and uniqueness fail only if there is some solution $u(t)$ such that $|D u(t)|$ becomes infinite in some finite time.

Theorem 47. Suppose that the 3D Navier-Stokes equations are well posed on $V$, so that for any $f \in H$ and $u_{0} \in V$,

$$
\frac{\mathrm{d} u}{\mathrm{~d} t}+A u+B(u, u)=f
$$

has a strong solution $u(t)$, that is, a solution $u$ with

$$
u \in L^{\infty}(0, T ; V) \cap L^{2}(0, T ; D(A))
$$

for all $T>0$. Then there exists an absorbing set in $V$.

The theorem is due to Constantin et al. [7]; the proof also appears in [13] and in Temam [18].

\section{Acknowledgment}

JCR is currently an EPSRC Leadership Fellow, grant EP/ G007470/1. This review paper is a substantially updated version of lectures given at the Instructional Conference on 'The Mathematical Analysis of Hydrodynamics' held at the ICMS in Edinburgh in June 2003; my thanks to the organisers of that meeting for their kind invitation.

\section{References}

[1] G. K. Batchelor, Introduction to Fluid Dynamics, Cambridge University Press, Cambridge, Mass, USA, 1967.
[2] C. R. Doering and J. D. Gibbon, Applied Analysis of the NavierStokes Equations, Cambridge Texts in Applied Mathematics, Cambridge University Press, Cambridge, Mass, USA, 1995.

[3] J. Leray, "Sur le mouvement d'un liquide visqueux emplissant l'espace," Acta Mathematica, vol. 63, no. 1, pp. 193-248, 1934.

[4] E. Hopf, "Über die Aufgangswertaufgave für die hydrodynamischen Grundliechungen," Mathematische Nachrichten, vol. 4, pp. 213-231, 1951.

[5] O. A. Ladyzhenskaya, The Mathematical Theory of Viscous Incompressible Flow, Gordon and Breach Science Publishers, New York, NY, USA, 1969.

[6] O. Ladyzhenskaya, Attractors for Semigroups and Evolution Equations, Cambridge University Press, Cambridge, Mass, USA, 1991.

[7] P. Constantin, C. Foias, and R. Temam, "Attractors representing turbulent flows," Memoirs of the American Mathematical Society, vol. 53, no. 314, 1985.

[8] J. K. Hale, L. T. Magalhães, and W. M. Oliva, Dynamics in Infinite Dimensions, vol. 47 of Applied Mathematical Sciences, Springer, New York, NY, USA, 2nd edition, 2002.

[9] P. G. Lemarié-Rieusset, Recent Developments in the NavierStokes Problem, vol. 431 of Chapman \& Hall/CRC Research Notes in Mathematics, Chapman \& Hall/CRC, Boca Raton, Fla, USA, 2002.

[10] M. Cannone, "Harmonic analysis tools for solving the incompressible Navier-Stokes equations," in Handbook of Mathematical Fluid Dynamics, S. Friedlander and D. Serre, Eds., vol. 3, pp. 161-244, North-Holland, Amsterdam, The Netherlands, 2004.

[11] U. Frisch, Turbulence, Cambridge University Press, Cambridge, Mass, USA, 1995.

[12] A. Cheskidov, C. R. Doering, and N. P. Petrov, "Energy dissipation in fractal-forced flow," Journal of Mathematical Physics, vol. 48, no. 6, Article ID 065208, 10 pages, 2007.

[13] P. Constantin and C. Foias, Navier-Stokes Equations, Chicago Lectures in Mathematics, University of Chicago Press, Chicago, Ill, USA, 1988.

[14] R. Temam, Navier-Stokes Equations: Theory and Numerical Analysis, vol. 2 of Studies in Mathematics and Its Applications, North-Holland Publishing, Amsterdam, The Netherlands, 1979.

[15] R. Temam, Navier-Stokes Equations and Nonlinear Functional Analysis, SIAM, Philadelphia, Pa, USA, 1985.

[16] J. C. Robinson, Infinite-Dimensional Dynamical Systems, Cambridge Texts in Applied Mathematics, Cambridge University Press, Cambridge, Mass, USA, 2001.

[17] L. C. Evans, Partial Differential Equations, vol. 19 of Graduate Studies in Mathematics, American Mathematical Society, Providence, RI, USA, 1998.

[18] R. Temam, Infinite-Dimensional Dynamical Systems in Mechanics and Physics, vol. 68 of Applied Mathematical Sciences, Springer, New York, NY, USA, 1988.

[19] I. Kukavica, "Log-log convexity and backward uniqueness," Proceedings of the American Mathematical Society, vol. 135, no. 8, pp. 2415-2421, 2007.

[20] J. Simon, "On the existence of the pressure for solutions of the variational Navier-Stokes equations," Journal of Mathematical Fluid Mechanics, vol. 1, no. 3, pp. 225-234, 1999.

[21] E. M. Stein, Harmonic Analysis: Real-Variable Methods, Orthogonality, and Oscillatory Integrals, vol. 43 of Princeton Mathematical Series, Princeton University Press, Princeton, NJ, USA, 1993.

[22] V. Shapiro, Fourier Series in Several Variables with Applications to Partial Differential Equations, Chapman \& Hall, 2011. 
[23] M. Larchevêque, "Équation de Monge-Ampère et écoulements incompressibles bidimensionnels," Comptes Rendus de l'Académie des Sciences. Série II, vol. 311, no. 1, pp. 33-36, 1990.

[24] D. Gilbarg and N. S. Trudinger, Elliptic Partial Differential Equations of Second Order, Springer, Berlin, Germany, 1998.

[25] C. Foias and J.-C. Saut, "Asymptotic behavior, as $t \rightarrow \infty$, of solutions of Navier-Stokes equations and nonlinear spectral manifolds," Indiana University Mathematics Journal, vol. 33, no. 3, pp. 459-477, 1984.

[26] M. E. Schonbek, "L ${ }^{2}$ decay for weak solutions of the NavierStokes equations," Archive for Rational Mechanics and Analysis, vol. 88, no. 3, pp. 209-222, 1985.

[27] T. Gallay and C. E. Wayne, "Invariant manifolds and the longtime asymptotics of the Navier-Stokes and vorticity equations on $\mathbb{R}^{2}$," Archive for Rational Mechanics and Analysis, vol. 163, no. 3, pp. 209-258, 2002.

[28] A. V. Babin and M. I. Vishik, Attractors of Evolution Equations, vol. 25 of Studies in Mathematics and Its Applications, NorthHolland Publishing Co., Amsterdam, The Netherlands, 1992.

[29] V. V. Chepyzhov and M. I. Vishik, Attractors for Equations of Mathematical Physics, vol. 49 of American Mathematical Society Colloquium Publications, American Mathematical Society, Providence, RI, USA, 2002.

[30] I. D. Chueshov, Introduction to the Theory of Infinite-Dimensional Dissipative Systems, ACTA Scientific Publishing House, Kharkiv, Ukraine, 2002.

[31] J. K. Hale, Asymptotic Behavior of Dissipative Systems, vol. 25 of Mathematical Surveys and Monographs, American Mathematical Society, Providence, RI, USA, 1988.

[32] M. I. Vishik, Asymptotic Behaviour of Solutions of Evolutionary Equations, Cambridge University Press, Cambridge, Mass, USA, 1992.

[33] H. Crauel, "Random point attractors versus random set attractors," Journal of the London Mathematical Society, vol. 63, no. 2, pp. 413-427, 2001.

[34] J. E. Billotti and J. P. LaSalle, "Dissipative periodic processes," Bulletin of the American Mathematical Society, vol. 77, pp. 1082$1088,1971$.

[35] B. Günther and J. Segal, "Every attractor of a flow on a manifold has the shape of a finite polyhedron," Proceedings of the American Mathematical Society, vol. 119, no. 1, pp. 321-329, 1993.

[36] R. H. Bing, "Concerning hereditarily indecomposable continua," Pacific Journal of Mathematics, vol. 1, pp. 43-51, 1951.

[37] J. A. Langa and J. C. Robinson, "Determining asymptotic behavior from the dynamics on attracting sets," Journal of Dynamics and Differential Equations, vol. 11, no. 2, pp. 319-331, 1999.

[38] B. M. Garay, "Strong cellularity and global asymptotic stability," Fundamenta Mathematicae, vol. 138, no. 2, pp. 147-154, 1991.

[39] J. C. Robinson and J. J. Sánchez-Gabites, "On finite-dimensional attractors of homeomorphisms," http://arxiv.org/abs/1306.5959.

[40] P. Constantin, C. Foias, I. Kukavica, and A. J. Majda, "Dirichlet quotients and 2D periodic Navier-Stokes equations," Journal de Mathématiques Pures et Appliquées, vol. 76, no. 2, pp. 125-153, 1997.

[41] J. C. Robinson, "Low dimensional attractors arise from forcing at small scales," Physica D, vol. 181, no. 1-2, pp. 39-44, 2003.

[42] J. D. Gibbon and G. A. Pavliotis, "Estimates for the two-dimensional Navier-Stokes equations in terms of the Reynolds number," Journal of Mathematical Physics, vol. 48, no. 6, Article ID 065202, 14 pages, 2007.
[43] C. R. Doering and C. Foias, "Energy dissipation in body-forced turbulence," Journal of Fluid Mechanics, vol. 467, pp. 289-306, 2002.

[44] C. Foias and G. Prodi, "Sur le comportement global des solutions non-stationnaires des équations de Navier-Stokes en dimension 2," Rendiconti del Seminario Matematico della Università di Padova, vol. 39, pp. 1-34, 1967.

[45] O. A. Ladyzhenskaya, "On the dynamical system generated by the Navier-Stokes equations," Journal of Soviet Mathematics, vol. 3, p. 4, 1975, English translation in Zapiskii Nauchnich Seminarovs LOMI, vol. 27, pp. 91-114, 1972.

[46] C. Foias and R. Temam, "Some analytic and geometric properties of the solutions of the evolution Navier-Stokes equations," Journal de Mathématiques Pures et Appliquées. Neuvième Série, vol. 58, no. 3, pp. 339-368, 1979.

[47] C. Foias, O. Manley, and R. Temam, "Modelling of the interaction of small and large eddies in two-dimensional turbulent flows," Mathematical Modelling and Numerical Analysis, vol. 22, no. 1, pp. 93-118, 1988.

[48] J. G. Heywood and R. Rannacher, "Finite element approximation of the nonstationary Navier-Stokes problem. I. Regularity of solutions and second-order error estimates for spatial discretization," SIAM Journal on Numerical Analysis, vol. 19, no. 2, pp. 275-311, 1982.

[49] C. Guillopé, “Comportement à l'infini des solutions des équations de Navier-Stokes et propriété des ensembles fonctionnels invariants (ou attracteurs)," Annales de l'Institut Fourier, vol. 32, no. 3, pp. 1-37, 1982.

[50] C. Foias and R. Temam, "Gevrey class regularity for the solutions of the Navier-Stokes equations," Journal of Functional Analysis, vol. 87, no. 2, pp. 359-369, 1989.

[51] F. John, Partial Differential Equations, vol. 1 of Applied Mathematical Sciences, Springer, New York, NY, USA, 4th edition, 1982.

[52] I. Kukavica, "On the time analyticity radius of the solutions of the two-dimensional Navier-Stokes equations," Journal of Dynamics and Differential Equations, vol. 3, no. 4, pp. 611-618, 1991.

[53] P. K. Friz and J. C. Robinson, "Parametrising the attractor of the two-dimensional Navier-Stokes equations with a finite number of nodal values," Physica D, vol. 148, no. 3-4, pp. 201-220, 2001.

[54] O. A. Ladyzhenskaya, "The finite-dimensionality of bounded invariant sets for the Navier-Stokes system and other dissipative systems," Zapiskii Nauchnich Seminarovs LOMI, vol. 115, pp. 137-155, 1982.

[55] A. Eden, C. Foias, B. Nicolaenko, and R. Temam, Exponential Attractors for Dissipative Evolution Equations, vol. 37 of RAM: Research in Applied Mathematics, Masson, Paris, France, 1994.

[56] K. J. Falconer, The Geometry of Fractal Sets, vol. 85 of Cambridge Tracts in Mathematics, Cambridge University Press, Cambridge, Mass, USA, 1986.

[57] K. Falconer, Fractal Geometry, John Wiley \& Sons, Chichester, UK, 1990.

[58] J. C. Robinson, Dimensions, Embeddings, and Attractors, vol. 186 of Cambridge Tracts in Mathematics, Cambridge University Press, Cambridge, Mass, USA, 2011.

[59] J. Mallet-Paret, "Negatively invariant sets of compact maps and an extension of a theorem of Cartwright," Journal of Differential Equations, vol. 22, no. 2, pp. 331-348, 1976.

[60] R. Mañé, "On the dimension of the compact invariant sets of certain nonlinear maps," in Dynamical Systems and Turbulence, 
Warwick 1980 (Coventry, 1979/1980), vol. 898 of Lecture Notes in Mathematics, pp. 230-242, Springer, Berlin, Germany, 1981.

[61] A. N. Carvalho, J. A. Langa, and J. C. Robinson, "Finite-dimensional global attractors in Banach spaces," Journal of Differential Equations, vol. 249, no. 12, pp. 3099-3109, 2010.

[62] P. Constantin and C. Foias, "Global Lyapunov exponents, Kaplan-Yorke formulas and the dimension of the attractors for 2D Navier-Stokes equations," Communications on Pure and Applied Mathematics, vol. 38, no. 1, pp. 1-27, 1985.

[63] A. N. Carvalho, J. A. Langa, and J. C. Robinson, Attractors for Infinite-Dimensional Non-Autonomous Dynamical Systems, Applied Mathematical Sciences 182, Springer, New York, NY, USA, 2012.

[64] A. Douady and J. Oesterlé, "Dimension de Hausdorff des attracteurs," Comptes Rendus Hebdomadaires des Séances de l'Académie des Sciences. Séries A et B, vol. 290, no. 24, pp. A1135A1138, 1980.

[65] V. V. Chepyzhov and A. A. Ilyin, "A note on the fractal dimension of attractors of dissipative dynamical systems," Nonlinear Analysis: Theory, Methods \& Applications, vol. 44, no. 6, pp. 811819, 2001.

[66] V. V. Chepyzhov and A. A. Ilyin, "On the fractal dimension of invariant sets: applications to Navier-Stokes equations," Discrete and Continuous Dynamical Systems, vol. 10, no. 1-2, pp. 117-135, 2004.

[67] E. Lieb and W. Thirring, "Inequalities for the moments of the eigenvalues of the Schrödinger equations and their relation to Sobolev inequalities," in Studies in Mathematical Physics: Essays in Honor of Valetine Bargmann, E. Lieb, B. Simon, and A. S. Wightman, Eds., pp. 269-303, Princeton University Press, Princeton, NJ, USA, 1976.

[68] P. Constantin, C. Foias, and R. Temam, "On the dimension of the attractors in two-dimensional turbulence," Physica D, vol. 30, no. 3, pp. 284-296, 1988.

[69] A. V. Babin and M. I. Vishik, Attractors of Evolution Equations, North Holland, Amsterdam, The Netherlands, 1992.

[70] V. X. Liu, "A sharp lower bound for the Hausdorff dimension of the global attractors of the 2D Navier-Stokes equations," Communications in Mathematical Physics, vol. 158, no. 2, pp. 327-339, 1993.

[71] M. Ziane, "Optimal bounds on the dimension of the attractor of the Navier-Stokes equations," Physica D, vol. 105, no. 1-3, pp. $1-19,1997$.

[72] E. Olson and E. S. Titi, "Determining modes for continuous data assimilation in 2D turbulence," Journal of Statistical Physics, vol. 113, no. 5-6, pp. 799-840, 2003.

[73] C. V. Tran and T. G. Shepherd, "Constraints on the spectral distribution of energy and enstrophy dissipation in forced twodimensional turbulence," Physica D, vol. 165, no. 3-4, pp. 199212, 2002.

[74] L. D. Landau and E. M. Lifshitz, Fluid Mechanics, vol. 6 of Course of Theoretical Physics, Pergamon Press, Oxford, UK, 1959.

[75] R. H. Kraichnan, "Inertial ranges in two-dimensional turbulence," Physics of Fluids, vol. 10, pp. 1417-1423, 1976.

[76] J. C. Robinson, "Parametrization of global attractors, experimental observations, and turbulence," Journal of Fluid Mechanics, vol. 578, pp. 495-507, 2007.

[77] B. R. Hunt and V. Yu. Kaloshin, "Regularity of embeddings of infinite-dimensional fractal sets into finite-dimensional spaces," Nonlinearity, vol. 12, no. 5, pp. 1263-1275, 1999.
[78] A. Ben-Artzi, A. Eden, C. Foias, and B. Nicolaenko, "Hölder continuity for the inverse of Mañés projection," Journal of Mathematical Analysis and Applications, vol. 178, no. 1, pp. 2229, 1993.

[79] C. Foias and E. Olson, "Finite fractal dimension and HölderLipschitz parametrization," Indiana University Mathematics Journal, vol. 45, no. 3, pp. 603-616, 1996.

[80] E. J. Olson and J. C. Robinson, "Almost bi-Lipschitz embeddings and almost homogeneous sets," Transactions of the American Mathematical Society, vol. 362, no. 1, pp. 145-168, 2010.

[81] E. Pinto de Moura and J. C. Robinson, "Lipschitz deviation and embeddings of global attractors," Nonlinearity, vol. 23, no. 7, pp. 1695-1708, 2010.

[82] B. R. Hunt, T. Sauer, and J. A. Yorke, "Prevalence: a translationinvariant almost every for infinite dimensional spaces," Bulletin of the American Mathematical Society, vol. 27, pp. 217-238, 1992.

[83] J. C. Robinson, "Linear embeddings of finite-dimensional subsets of Banach spaces into Euclidean spaces," Nonlinearity, vol. 22, no. 4, pp. 711-728, 2009.

[84] P. K. Friz and J. C. Robinson, "Smooth attractors have zero 'thickness"' Journal of Mathematical Analysis and Applications, vol. 240, no. 1, pp. 37-46, 1999.

[85] W. Ott, B. Hunt, and V. Kaloshin, "The effect of projections on fractal sets and measures in Banach spaces," Ergodic Theory and Dynamical Systems, vol. 26, no. 3, pp. 869-891, 2006.

[86] J. Heinonen, Lectures on Analysis on Metric Spaces, Universitext, Springer, New York, NY, USA, 2001.

[87] T. Sauer, J. A. Yorke, and M. Casdagli, "Embedology," Journal of Statistical Physics, vol. 65, no. 3-4, pp. 579-616, 1991.

[88] J. C. Robinson, "A topological delay embedding theorem for infinite-dimensional dynamical systems," Nonlinearity, vol. 18, no. 5, pp. 2135-2143, 2005.

[89] C. Foias and R. Temam, "Determination of the solutions of the Navier-Stokes equations by a set of nodal values," Mathematics of Computation, vol. 43, no. 167, pp. 117-133, 1984.

[90] C. Foias and E. S. Titi, "Determining nodes, finite difference schemes and inertial manifolds," Nonlinearity, vol. 4, no. 1, pp. 135-153, 1991.

[91] D. A. Jones and E. S. Titi, "On the number of determining nodes for the 2D Navier-Stokes equations," Journal of Mathematical Analysis and Applications, vol. 168, no. 1, pp. 72-88, 1992.

[92] D. A. Jones and E. S. Titi, "Upper bounds on the number of determining modes, nodes, and volume elements for the Navier-Stokes equations," Indiana University Mathematics Journal, vol. 42, no. 3, pp. 875-887, 1993.

[93] B. Cockburn, D. A. Jones, and E. S. Titi, "Estimating the number of asymptotic degrees of freedom for nonlinear dissipative systems," Mathematics of Computation, vol. 66, no. 219, pp. 1073-1087, 1997.

[94] I. Kukavica and J. C. Robinson, "Distinguishing smooth functions by a finite number of point values, and a version of the Takens embedding theorem," Physica D, vol. 196, no. 1-2, pp. 4566, 2004.

[95] C.-C. Poon, "Unique continuation for parabolic equations," Communications in Partial Differential Equations, vol. 21, no. 34, pp. 521-539, 1996.

[96] C.-C. Poon, "Blow-up behavior for semilinear heat equations in nonconvex domains," Differential and Integral Equations, vol. 13, no. 7-9, pp. 1111-1138, 2000.

[97] C. Foias, O. P. Manley, R. Temam, and Y. M. Trève, "Asymptotic analysis of the Navier-Stokes equations," Physica D, vol. 9, no. 1-2, pp. 157-188, 1983. 
[98] D. A. Jones and E. S. Titi, "Determining finite volume elements for the 2D Navier-Stokes equations," Physica D, vol. 60, no. 1-4, pp. 165-174, 1992.

[99] C. Foias, G. R. Sell, and R. Temam, "Variétés inertielles des équations différentielles dissipatives," Comptes Rendus des Séances de l'Académie des Sciences. Série I, vol. 301, no. 5, pp. 139-141, 1985.

[100] C. Foias, G. R. Sell, and R. Temam, "Inertial manifolds for nonlinear evolutionary equations," Journal of Differential Equations, vol. 73, no. 2, pp. 309-353, 1988.

[101] M. Renardy and R. C. Rogers, An Introduction to Partial Differential Equations, vol. 13 of Texts in Applied Mathematics, Springer, New York, NY, USA, 1992.

[102] P. Hartman, Ordinary Differential Equations, John Wiley \& Sons, New York, NY, USA, 1964.

[103] C. Foias, B. Nicolaenko, G. R. Sell, and R. Temam, "Inertial manifolds for the Kuramoto-Sivashinsky equation and an estimate of their lowest dimension," Journal de Mathématiques Pures et Appliquées, vol. 67, no. 3, pp. 197-226, 1988.

[104] R. Temam and X. M. Wang, "Estimates on the lowest dimension of inertial manifolds for the Kuramoto-Sivashinsky equation in the general case," Differential and Integral Equations, vol. 7, no. 3-4, pp. 1095-1108, 1994.

[105] J. Mallet-Paret and G. R. Sell, "Inertial manifolds for reaction diffusion equations in higher space dimensions," Journal of the American Mathematical Society, vol. 1, no. 4, pp. 805-866, 1988.

[106] P. Collet, J.-P. Eckmann, H. Epstein, and J. Stubbe, "A global attracting set for the Kuramoto-Sivashinsky equation," Communications in Mathematical Physics, vol. 152, no. 1, pp. 203-214, 1993.

[107] E. Pinto de Moura and J. C. Robinson, "Log-Lipschitz continuity of the vector field on the attractor of certain parabolic equations," http://arxiv.org/abs/1008.4949.

[108] J. H. Wells and L. R. Williams, Embeddings and Extensions in Analysis, Springer, New York, NY, USA, 1975.

[109] J. C. Robinson, "Global attractors: topology and finite-dimensional dynamics," Journal of Dynamics and Differential Equations, vol. 11, no. 3, pp. 557-581, 1999.

[110] A. V. Romanov, "Finite-dimensional limit dynamics of dissipative parabolic equations," Matematicheskiǔ Sbornik, vol. 191, no. 3, pp. 99-112, 2000.

[111] A. Eden, V. Kalantarov, and S. Zelik, "Counterexamples to the regularity of Mane projections and global attractors," to appear in Russian Mathematical Surveys, http://arxiv.org/abs/1108.0217.

[112] E. Pinto de Moura, J. C. Robinson, and J. J. Sánchez-Gabites, "Embedding of global attractors and their dynamics," Proceedings of the American Mathematical Society, vol. 139, no. 10, pp. 3497-3512, 2011. 


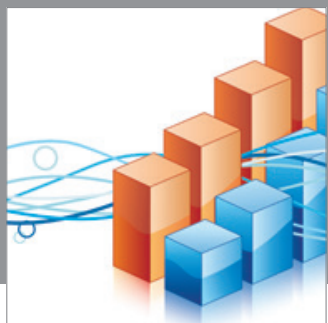

Advances in

Operations Research

mansans

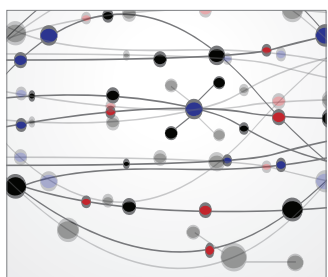

The Scientific World Journal
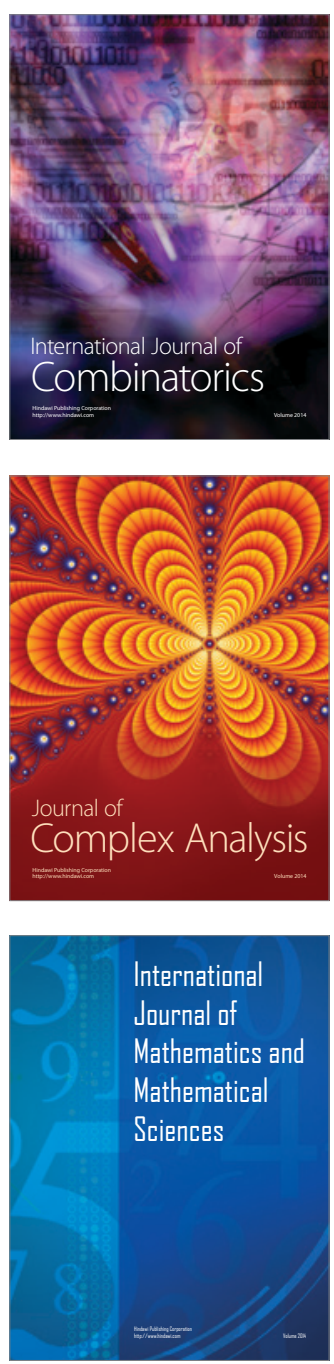
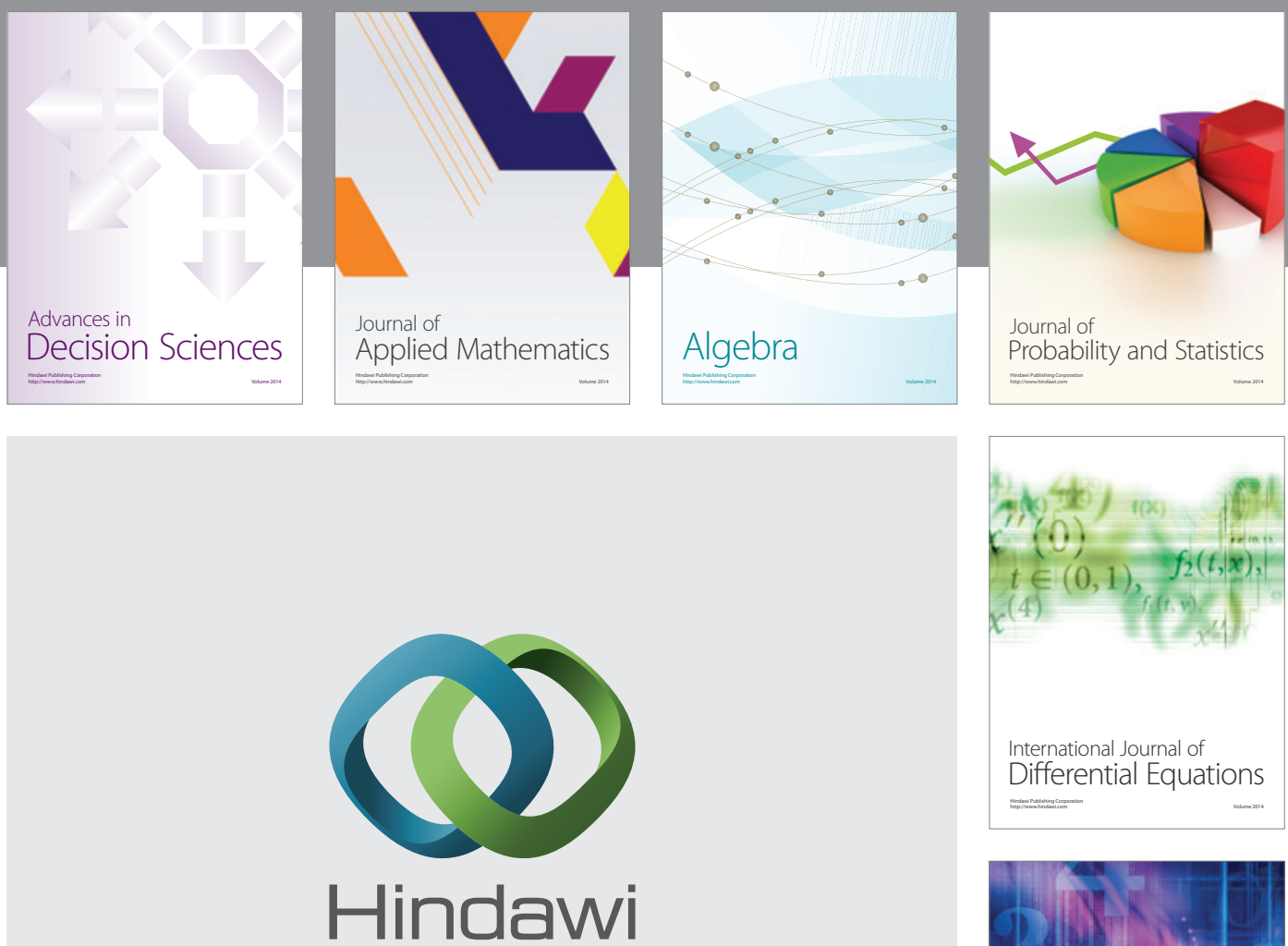

Submit your manuscripts at http://www.hindawi.com
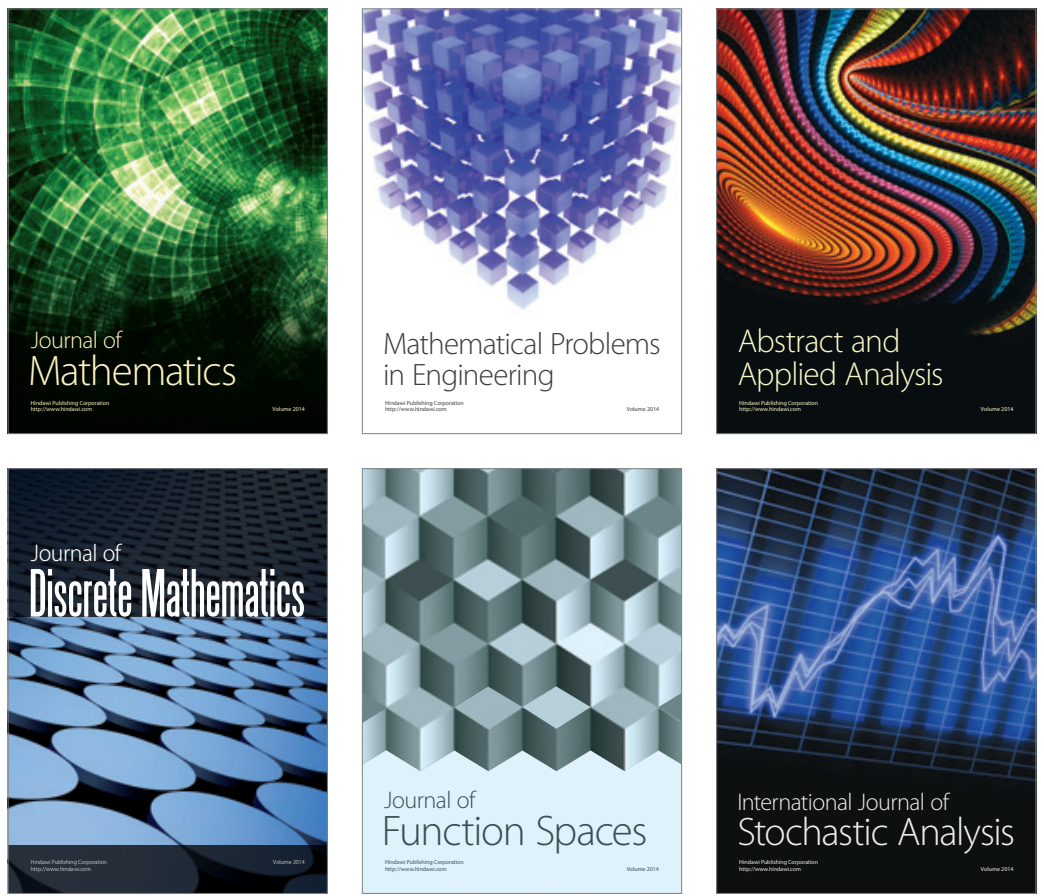

Journal of

Function Spaces

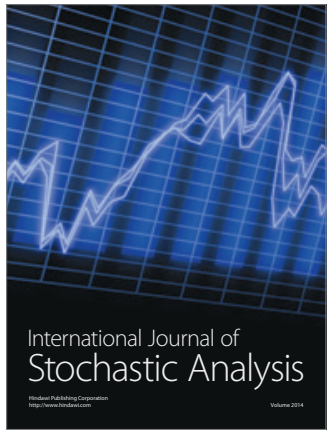

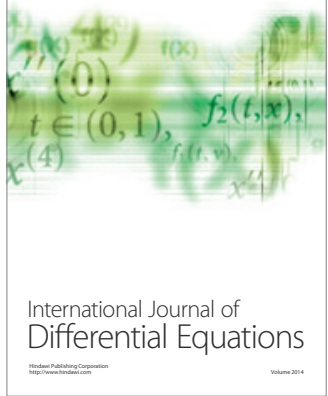
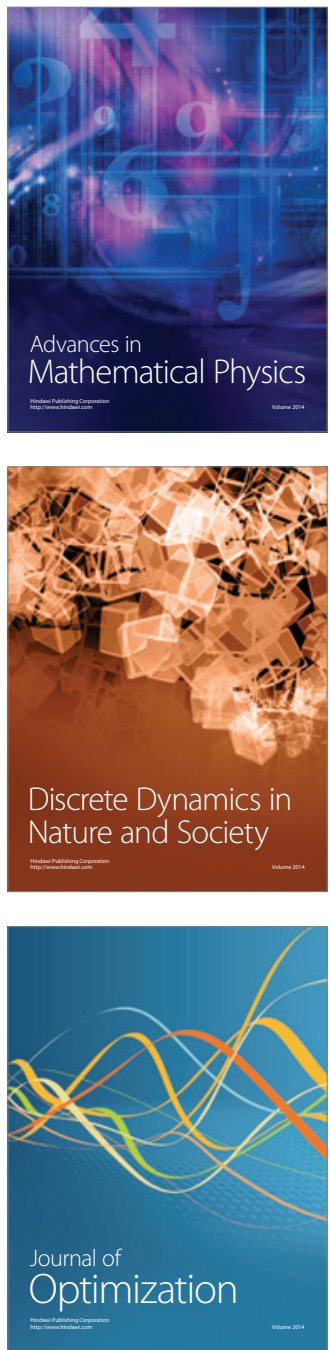